Draft version April 20, 2012

Preprint typeset using $\mathrm{LAT}_{\mathrm{E}} \mathrm{X}$ style emulateapj v. 11/10/09

\title{
A MISMATCH IN THE ULTRAVIOLET SPECTRA BETWEEN LOW-REDSHIFT AND INTERMEDIATE-REDSHIFT TYPE IA SUPERNOVAE AS A POSSIBLE SYSTEMATIC UNCERTAINTY FOR SUPERNOVA COSMOLOGY
}

Ryan J. Foley ${ }^{1,2,3}$, Alexei V. Filippenko ${ }^{2}$, Richard Kessler $^{4,5}$, Bruce Bassett ${ }^{6,7,8}$, Joshua A. Frieman ${ }^{4,5,9}$, Peter M. Garnavich ${ }^{10}$, Saurabh W. Jha ${ }^{11}$, Kohki Konishi ${ }^{12}$, Hubert Lampeitl ${ }^{13}$, Adam G. Riess ${ }^{14,15}$, Masao Sako ${ }^{16}$, Donald P. Schneider ${ }^{17,18}$, Jesper Sollerman ${ }^{19}$, And Mathew Smith ${ }^{6}$

Draft version April 20, 2012

\begin{abstract}
We present Keck high-quality rest-frame ultraviolet (UV) through optical spectra of 21 Type Ia supernovae (SNe Ia) in the redshift range $0.11 \leq z \leq 0.37$ and a mean redshift of 0.22 that were discovered during the Sloan Digital Sky Survey-II (SDSS-II) SN Survey. Using the broad-band photometry of the SDSS survey, we are able to reconstruct the SN host-galaxy spectral energy distributions (SEDs), allowing for a correction for the host-galaxy contamination in the SN Ia spectra. Comparison of composite spectra constructed from a subsample of 17 high-quality spectra to those created from a low-redshift sample with otherwise similar properties shows that the Keck/SDSS SNe Ia have, on average, extremely similar rest-frame optical spectra but show a UV flux excess. This observation is confirmed by comparing synthesized broad-band colors of the individual spectra, showing a difference in mean colors at the 2.4-4.4 $\sigma$ level for various UV colors. We further see a slight difference in the UV spectral shape between SNe with low-mass and high-mass host galaxies. Additionally, we detect a relationship between the flux ratio at 2770 and $2900 \AA$ and peak luminosity that differs from that observed at low redshift. We find that changing the UV SED of an SN Ia within the observed dispersion can change the inferred distance moduli by $\sim 0.1 \mathrm{mag}$. This effect only occurs when the data probe the rest-frame UV. We suggest that this discrepancy could be due to differences in the host-galaxy population of the two SN samples or to small-sample statistics.
\end{abstract}

Subject headings: supernovae - general, cosmology —observations, distance scale

1 Harvard-Smithsonian Center for Astrophysics, 60 Garden Street, Cambridge, MA 02138, USA.

2 Department of Astronomy, University of California, Berkeley, CA 94720-3411, USA.

3 Clay Fellow. Electronic address rfoley@cfa.harvard.edu .

4 Kavli Institute for Cosmological Physics, The University of Chicago, 5640 South Ellis Avenue, Chicago, IL 60637, USA.

5 Department of Astronomy and Astrophysics, The University of Chicago, 5640 South Ellis Avenue, Chicago, IL 60637.

6 Department of Mathematics and Applied Mathematics, University of Cape Town, Rondebosch 7701, South Africa.

7 South African Astronomical Observatory, P.O. Box 9, Observatory 7935, South Africa.

8 African Institute for Mathematical Sciences, 6-8 Melrose

Road, Muizenberg, Cape Town, South Africa.

9 Center for Particle Astrophysics, Fermi National Accelerator Laboratory, P.O. Box 500, Batavia, IL 60510, USA.

10 University of Notre Dame, 225 Nieuwland Science, Notre Dame, IN 46556-5670, USA.

11 Department of Physics and Astronomy, Rutgers University, 136 Frelinghuysen Road, Piscataway, NJ 08854, USA.

12 Institute for Cosmic Ray Research, University of Tokyo,

5-1-5, Kashiwanoha, Kashiwa, Chiba, 277-8582, Japan.

13 Institute of Cosmology and Gravitation, Mercantile House Hampshire Terrace, University of Portsmouth, Portsmouth PO1 2EG, UK.

${ }^{14}$ Department of Physics and Astronomy, Johns Hopkins University, Baltimore, MD 21218, USA.

15 Space Telescope Science Institute, 3700 San Martin Drive, Baltimore, MD 21218, USA.

16 Department of Physics and Astronomy, University of Pennsylvania, 209 South 33rd Street, Philadelphia, PA 19104, USA.

17 Department of Astronomy and Astrophysics, 525 Davey Laboratory, Pennsylvania State University, University Park, PA 16802, USA.

18 Institute for Gravitation and the Cosmos, The Pennsylvania State University, University Park, PA 16802, USA.

19 Oskar Klein Centre, Department of Astronomy, Stockholm University, 10691 Stockholm, Sweden. 


\section{INTRODUCTION}

Type Ia supernovae (SNe Ia), being both extremely luminous and having well-calibrated peak luminosities (starting with the work of Phillips 1993), are excellent and precise distance indicators at cosmological scales; see, for example, Leibundgut (2001), Livio (2001), Perlmutter \& Schmidt (2003), and Filippenko (2005b) for reviews. Measurements of several hundred SNe Ia at low redshifts (e.g., Hamuv et al. 1996; Riess et al. 1999; Jha et al. 2006; Hicken et al. 2009a; Contreras et al. 2010; Ganeshalingam et al. 2010; Stritzinger et al.|2011) and high redshifts have shown that the expansion rate of the Universe is currently accelerating (Riess et al. 1998; Perlmutter et al. 1999), and that the equationof-state parameter of the dark energy, $w=P /\left(\rho c^{2}\right)$, is consistent with the cosmological constant, $w=$ -1 (e.g., Riess et al. 2007; Wood-Vasey et al. 2007; Hicken et al. 2009b; Kessler et al. 2009a; Conley et al. 2011; Sullivan et al. 2011). The underlying assumption of this work is that we can accurately measure distances to $\mathrm{SNe}$ Ia at high redshifts using relationships between distances and observations of SNe Ia at low redshifts.

If there are physical differences between low-redshift and high-redshift SNe Ia (in a way that affects our derived luminosity distances), we would expect to notice discrepancies in their observed spectra. Theoretical studies have determined that changing progenitor metallicity, a likely difference between low-redshift and high-redshift SNe Ia, produces relatively small variations in the optical spectra of SNe Ia but larger differences in their ultraviolet (UV) spectra (e.g., Höflich et al. 1998; Lentz et al. 2000; Sauer et al. 2008). Foley et al. (2008a) and Ellis et al. (2008) have recently shown that high-redshift SNe Ia are similar to their low-redshift counterparts, though both studies had major limitations: an inability to properly correct for host-galaxy contamination in the highredshift SN spectra (Foley et al. 2008a) and an insufficient low-redshift comparison sample (Ellis et al. 2008).

Sullivan et al. (2009) compared composite spectra from three samples of SNe Ia with mean redshifts $(z)$ of $0.02,0.48$, and 1.16 . The spectra in the samples had (by construction) similar phases, but the SNe had slightly different stretches (consistent with the trend with redshift; Howell et al. 2007) and colors. The composite spectra differed such that the strength of features corresponding to intermediate-mass elements (IMEs) are weaker at higher redshifts. Sullivan et al. (2009) argue that the changing stretch distribution with redshift results in SN Ia explosions producing more ${ }^{56} \mathrm{Ni}$, and thus having less mass in IMEs, at higher redshift. Sullivan et al. (2009) also found that their low-redshift composite spectrum had less flux for $\lambda<3500 \AA$, but they dismiss the result since their low-redshift sample contains only three $\mathrm{SNe}$ Ia with spectra covering these wavelengths. Cooke et al. (2011) created a composite spectrum from 10 nearby low-redshift $\mathrm{SNe}$ Ia and also found that the low-redshift SNe had less flux than the high-redshift SNe in the UV. Both the low and high-redshift samples had similar light-curve shape distributions, making it unlikely that the discrepancy is caused by differences in the population. Nonetheless, they decided not to examine this feature until they obtained more data.

Further indications of a possible change in the
UV properties of SNe Ia come from the analysis of Kessler et al. (2009a, hereafter K09), who found differences in the rest-frame $U$-band data for low-redshift and high-redshift samples. Using only the Sloan Digital Sky Survey-II (SDSS-II) sample to extract cosmological parameters, exclusion of the rest-frame UV region caused a systematic offset in the measurement of $w$ by $\sim 0.3$. (However, this offset is significantly reduced when combining with higher-redshift samples.) This difference is referred to as the " $U$-band anomaly" by K09. The differences could be caused by calibrations (the observer-frame $U$ band is notoriously difficult to properly calibrate), or by real differences in the samples. Since the inclusion of the $U$-band and SDSS $u$-band data had a very different effect on the low-redshift sample and the lowest-redshift SDSS-II SNe $(z<0.1)$, respectively, K09 suggested that the low-redshift, non-SDSS-II sample was the cause of the effect, which could easily be the result of incorrect calibration.

Foley, Filippenko, \& Jha (2008b, hereafter FFJ) showed that for a sample of six low-redshift SNe Ia, the steepness of their UV spectra near maximum brightness, the "UV ratio" $\left[\mathcal{R}_{U V}=f_{\lambda}(2770 \AA) / f_{\lambda}(2900 \AA)\right]$, appears to be highly correlated with peak luminosity. Although this relationship could provide new insights into the UV properties of SNe Ia and reduce the scatter in their calibrated absolute luminosities, it is based on few SNe (but additional low-redshift SNe Ia are consistent with the relationship; Bufano et al. 2009; Folev et al. 2012). Moreover, the relationship has not yet been tested at high redshifts.

The SDSS-II SN survey has discovered approximately 1000 SNe Ia at $z \lesssim 0.4$ with $\sim 500$ being spectroscopically confirmed (Frieman et al. 2008; Sako et al. 2008; Kessler et al. 2009a; Sollerman et al. 2009; Lampeitl et al. 2010a; Sako et al. 2011). We describe this redshift range as "intermediate," but note that if one must choose a binary description, it would naturally fall in the "high-redshift" category rather than the "low-redshift" one. As a complementary program at the Keck Observatory to examine potential differences in the UV spectral-energy distributions (SEDs) of lowredshift and high-redshift SNe Ia and to test the validity of the FFJ relationship at high redshift, we observed 21 SDSS-II SNe Ia (the "Keck/SDSS" sample) with the intention of obtaining high signal-to-noise ratio $(\mathrm{S} / \mathrm{N})$ rest-frame UV through optical spectra. We have used the deep multi-band photometry of the SN host galaxies from SDSS to correct for host-galaxy contamination. Utilizing the large, high quality, low-redshift SN Ia samples from Foley et al. (2008a) (described in greater detail by Foley et al. 2008a and FFJ), we have been able to perform a better comparison of low and high-redshift SN Ia spectra. Note that the low-redshift composite spectra presented by Foley et al. (2008a) were created using the same methods as those employed in this paper. Hsiao et al. (2007) also constructed a template spectrum similar to our low-redshift composite spectra (Foley et al. 2008a); however, the Hsiao et al. (2007) template spectra contain high-redshift SN Ia data and hence are not ideal for evolutionary studies.

This paper is structured in the following way. Section 2 discusses our sample of SNe Ia, the Keck spectroscopic 
observations, and the data reduction. We also describe how we correct for host-galaxy contamination and our method for creating composite spectra. In Section 3 , we compare our Keck/SDSS sample to the low-redshift sample presented by Foley et al. (2008a). Section 4 explores the implications of our findings for SN cosmology. In Section 5. we split the Keck/SDSS sample by various observational properties to determine their effect on spectra. We examine the UV ratio in Section 6. Section 7 explores potential systematic errors. We summarize our conclusions in Section 8, including some discussion for future research.

\section{OBSERVATIONS AND DATA REDUCTION}

The SDSS-II survey was a three-year extension of the SDSS (for technical descriptions of the SDSS and its data products, see York et al. 2000 and Stoughton et al. 2002). The SDSS-II SN Survey, one component of the SDSS-II program, was carried out over three seasons, each of duration three months (Sep.-Nov. 2005-2007). Using the SDSS wide-field imaging camera (Gunn et al. 1998) on the SDSS $2.5 \mathrm{~m}$ telescope (Gunn et al. 2006), it was a time-domain survey in ugriz over 300 square degrees centered on the celestial equator (Fukugita et al. 1996). The SN survey took advantage of the excellent photometric and astrometric calibration achieved by the SDSS (Hogg et al. 2001; Smith et al. 2002; Pier et al. 2003; Ivezić et al. 2004; Tucker et al. 2006). Its main purpose was to fill in the "redshift desert" $(0.1<z<0.3)$ in the existing SN Ia Hubble diagram (which was visible in SN Ia Hubble diagrams at the time of the creation of the project; e.g., Riess et al. 2004), with a focus on reducing systematic errors in SN Ia distance estimates. In total, the SDSS-II SN Survey discovered 500 spectroscopically confirmed SNe Ia out to a redshift of $\sim 0.4$ (Frieman et al. 2008). The first cosmological results were presented by K09, with additional investigations by Sollerman et al. (2009) and Lampeitl et al. (2010a).

$\mathrm{SNe}$ for the Keck/SDSS sample were detected as part of the SDSS-II SN survey (Barentine et al. 2005; Bassett et al. 2006, 2007a,b, c). In addition to many spectroscopic observations of SNe with smaller telescopes (Zheng et al. 2008; Ostman et al. 2011; Konishi et al. 2011), a specific program was developed at the Keck Observatory to observe the rest-frame UV spectra of $z \approx 0.3$ $\mathrm{SNe}$ Ia. Targets were selected in a manner described by Sako et al. (2008); objects which appeared to have the appropriate colors and light curves of an SN Ia near maximum light at $z \approx 0.3$ were chosen for observation in our program. Some host galaxies had predetermined redshifts, which would occasionally influence the decision to observe an object. About half of the targets were already spectroscopically confirmed as SNe Ia near maximum brightness in the appropriate redshift range by other telescopes before observations were made at Keck. No additional data (e.g., host-galaxy properties, brightness, etc.) were used in deciding which objects were observed.

\subsection{Photometry}

Precise SDSS-II SN photometry was obtained using the "Scene Modeling Photometry" (SMP) technique, developed for this purpose and described in detail by Holtzman et al. (2008). The basic idea of SMP is to model the data as a time-varying point source (the SN) and sky background along with a time-independent galaxy background, all convolved with a time-varying point-spread function (PSF). The model is constrained by a global fit that uses all of the images taken during the survey that cover the SN region. Each image is modeled as a sum of fluxes from the SN, host galaxy, sky background, and calibration stars near the SN. The calibration stars are taken from the SDSS catalog produced by Ivezić et al. (2007). The fitted parameters are SN position, SN flux for each epoch and passband, and the host-galaxy intensity distribution in each passband. The galaxy model for each passband is a $20 \times 20$ grid (with a grid scale set by the CCD pixel scale, $0 . .4 \times 0 . . " 4)$ in sky coordinates, and each of the $400 \times 5=2000$ intensities is an independent fit parameter. In addition to the fit parameters, the flux model also depends on measured quantities that include sky background, magnitudes of calibration stars, position-dependent PSF, and a scaling between calibrated flux and CCD counts. There is no pixel resampling or image convolution, resulting in correct statistical uncertainties.

\subsection{Spectroscopy}

Spectra of SDSS-II targets were obtained with the Keck-I $10 \mathrm{~m}$ telescope using the low-resolution imaging spectrometer (LRIS; Oke et al. 1995). Observations in 2005 were obtained with the $600 / 4000$ grism and the $400 / 8500$ grating with the polarimetry optics in place. Observations in 2006 and 2007 were obtained with the $400 / 3400$ grism without the polarimetry optics, resulting in better response at blue wavelengths. Additionally, an atmospheric dispersion compensator (ADC) was in place for observations in 2007. This resulted in spectra covering the entire optical range, approximately 3400 $9400 \AA$ A. All spectra were obtained at relatively low airmass, and several standard stars were observed at similar airmass throughout the night. To reduce differential slit losses due to atmospheric dispersion (Filippenko 1982), the spectra were obtained with a position angle near parallactic, except for some objects in 2007 which were observed at low airmass and with the ADC.

Additional spectra of a subset of the host galaxies were obtained with the MagE spectrograph (Marshall et al. 2008) on the Magellan Clay 6.5 m telescope in December 2008, well after the SNe had faded. The slit was placed at the position of the SN with a position angle that went through the host nucleus. Because of this alignment, we were able to extract spectra at both positions.

Standard CCD processing and spectrum extraction of the LRIS data were accomplished with IRAF 20 . The data were extracted using the optimal algorithm of Horne (1986). Low-order polynomial fits to calibration-lamp spectra were used to establish the wavelength scale. Small adjustments derived from night-sky lines in the object frames were applied. For the MagE spectra, the sky was subtracted from the images using the method described by Kelson (2003). We employed IRAF and

20 IRAF: the Image Reduction and Analysis Facility is distributed by the National Optical Astronomy Observatory, which is operated by the Association of Universities for Research in Astronomy, Inc. (AURA) under cooperative agreement with the National Science Foundation (NSF). 
our own routines to flux calibrate the data and remove telluric lines using the well-exposed continua of the spectrophotometric standards (Wade \& Horne 1988; Folev et al. 2003, 2009). We used the spectrophotometric standards BD+284211 and Feige 34 (Oke 1990) to calibrate the spectra from the blue arm and BD+174708 and HD 19445 (Oke \& Gunn 1983) to calibrate the red arm. Our relative photometric flux calibration is typically accurate to $5 \%$ across our entire wavelength range (Matheson et al. 2008; Silverman et al., submitted).

The Keck/SDSS sample presented in this paper consists of $21 \mathrm{SNe}$ Ia. The properties of the SNe and their spectra are listed in Table 1. Two individual spectra are presented in Figure 11 (see the online version for all individual spectra). Histograms of the sample's redshift, rest-frame phase $t$, and $\Delta^{21}$ are presented in Figure 2 It is worth noting that the low and high-redshift light curves were fit with different versions of the multicolor light-curve shape method (MLCS; Riess et al. 1996): at low redshift by the publicly available version detailed by Jha et al. (2007), and at high redshift by a slightly modified internal SDSS-II version. The differences between the implementations are minor, however, and the derived values are essentially the same.

Although all SNe in our sample are obviously SNe Ia based on spectral features (e.g., Filippenko 1997), we have also performed the Supernova Identification (SNID) procedure of Blondin \& Tonry (2007) for each spectrum, confirming our classifications. Heliocentric redshifts were determined through either template matching (with SNID) or from host-galaxy emission or absorption lines, with the precision depending on the method (template matching vs. narrow host-galaxy lines) and the resolution of the spectrum from which the redshift is measured. Redshifts determined from template matching, from narrow lines in the Keck spectra, and narrow lines in either the SDSS main sample or Magellan host-galaxy spectra correspond to redshifts with 2,3 , and 4 significant digits in Table 1 and uncertainties of $\sim 0.01,0.001$, and 0.0001 , respectively.

The spectra are typically of higher quality than most of those previously published for high-redshift SNe Ia. The single exception is SN 16789, which has a relatively low $\mathrm{S} / \mathrm{N}$ and has been removed from our final sample for analysis (but its host will be discussed later). There is one outlier (compared to the rest of the sample) in both redshift and light-curve shape: SN 7147. It is the lowest redshift $\mathrm{SN}(z=0.111)$, and separated in redshift space from the rest of the sample (the next lowest redshift is $0.180)$. It also has a distinct light curve; unlike the rest of the sample, which clusters near $\Delta=-0.2$, SN 7147 has $\Delta=0.36$, corresponding to a relatively fast-declining light curve and indicating a lower-luminosity SN. Its spectrum also supports a lower luminosity, with a "silicon ratio" of $\mathcal{R}(\mathrm{Si})=0.4$, corresponding to lower-luminosity "Branch-normal SNe Ia" (Nugent et al. 1995).

${ }^{21} \Delta$ is a unitless parameter that describes light-curve shape and is related to luminosity, with smaller values of $\Delta$ corresponding higher luminosity; $M_{V}(t=0)=-19.504+0.736 \Delta+$ $0.182 \Delta^{2}+5 \log \left(H_{0} / 65 \mathrm{~km} \mathrm{~s}^{-1} \mathrm{Mpc}^{-1}\right)$ mag; $\Delta=0$ corresponds to $\Delta m_{15}(B)=1.07 \mathrm{mag}$ and stretch $s_{B}=0.96$ (Riess et al. 1996; Jha et al. 2007).
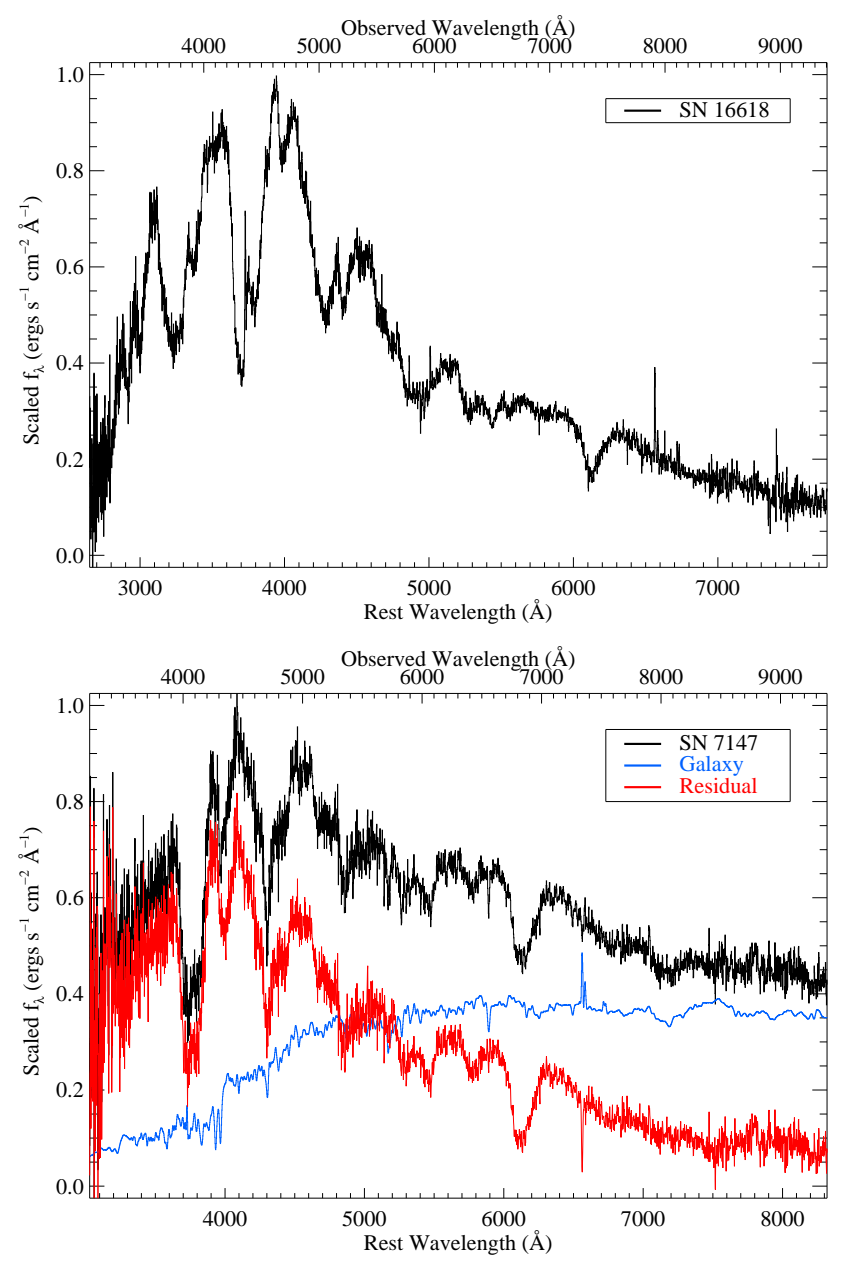

FIG. 1.- Spectra of SNe 16618 (top) and 7147 (bottom), examples of SNe with no and some host-galaxy contamination, respectively. The black curves are the observed spectra. The blue curve for SN 7147 is the reconstructed galaxy spectrum of its host. The red curve is the residual spectrum after galaxy-contamination correction. The galaxy-subtraction method produces clean SN spectra. See the electronic version for figures of all spectra of SNe Ia used in this study.

\subsection{Galaxy Subtraction}

As seen in Foley et al. (2008a), despite careful spectral reductions which attempt to remove host-galaxy light, many high-redshift SN Ia spectra have significant hostgalaxy contamination. Consequently, the intrinsic SN SED is difficult to recover from the spectrum. However, since the parameter space of galaxy SEDs is well known (and well behaved), one can reliably reconstruct galaxy SEDs with broad-band photometry (e.g., Blanton et al. 2003).

Adopting the approach described by Blanton et al. (2003), but updated to include UV wavelengths by Blanton \& Roweis (2007), and implemented in the IDL software package kcorrect.v4_1_422, we have used the ugriz photometry of the host galaxy at the position of the SN, which is a byproduct of the SMP method (Holtzman et al. 2008), and the redshifts presented in Table1 to reconstruct, using a $\chi^{2}$ minimization method,

22 http://howdy.physics.nyu.edu/index.php/Kcorrect . 
TABLE 1

SUPERNOVA INFORMATION

\begin{tabular}{|c|c|c|c|c|c|c|c|c|c|c|}
\hline $\begin{array}{c}\text { IAU SN } \\
\text { Name }\end{array}$ & $\begin{array}{l}\text { SDSS-II } \\
\text { ID }\end{array}$ & $\begin{array}{l}\text { UT Date of Spectrum } \\
\text { yyyy-mm-dd }\end{array}$ & Redshift & Airmass & $\begin{array}{c}\text { Galaxy } \\
\text { Cont. }(\%)^{\mathrm{a}}\end{array}$ & $\begin{array}{c}\text { Rest-Frame } \\
\text { Phase }(\text { days) }\end{array}$ & $\Delta^{\mathrm{c}}$ & $\begin{array}{l}\text { Host } A_{V} \\
(\mathrm{mag})^{\mathrm{d}}\end{array}$ & $\begin{array}{l}\text { Host Mass } \\
\left(10^{9} \mathrm{M}_{\odot}\right)^{\mathrm{e}}\end{array}$ & $\begin{array}{l}\text { Host } \\
B 1000^{\mathrm{f}}\end{array}$ \\
\hline $2005 \mathrm{jk}$ & 6304 & 2005-11-05.521 & 0.19 & 1.55 & 0.0 & $6.3(0.4)$ & $-0.06(0.11)$ & $0.41(0.06)$ & 20. & 0.33 \\
\hline $2005 i x$ & 6315 & $2005-11-05.253$ & 0.26 & 1.19 & 30.1 & $2.9(0.6)$ & $-0.15(0.08)$ & $0.01(0.02)$ & 1.7 & 3.2 \\
\hline $2005 \mathrm{jd}$ & 6649 & $2005-11-05.563$ & 0.32 & 1.83 & 1.6 & $2.7(0.5)$ & $-0.26(0.09)$ & $0.02(0.02)$ & 4.4 & 52 \\
\hline $2005 \mathrm{ik}$ & 6699 & 2005-11-05.292 & 0.32 & 1.20 & 0.0 & $3.0(0.6)$ & $-0.26(0.13)$ & $0.06(0.20)$ & 3.6 & 55 \\
\hline $2005 \mathrm{jc}$ & 6933 & $2005-11-05.478$ & 0.215 & 1.54 & 4.5 & $0.8(0.3)$ & $-0.17(0.09)$ & $0.22(0.07)$ & & \\
\hline $2005 \mathrm{jl}$ & 6936 & $2005-11-05.269$ & 0.180 & 1.14 & 7.4 & $-0.1(0.3)$ & $-0.20(0.08)$ & $0.20(0.06)$ & 7.0 & 4.1 \\
\hline $2005 \mathrm{jh}$ & 7147 & $2005-11-05.418$ & 0.111 & 1.60 & 32.3 & $0.0(0.2)$ & $0.36(0.07)$ & $0.01(0.02)$ & 12. & 5.6 \\
\hline $2005 \mathrm{jm}$ & 7243 & 2005-11-05.319 & 0.204 & 1.25 & 13.3 & $-4.6(0.1)$ & $-0.22(0.11)$ & $0.17(0.08)$ & 0.31 & 46 \\
\hline $2005 \mathrm{ji}$ & 7473 & $2005-11-05.436$ & 0.214 & 1.37 & 5.6 & $1.0(0.4)$ & $-0.07(0.09)$ & $0.02(0.03)$ & 2.0 & 67 \\
\hline 2005 jn & 7475 & $2005-11-05.457$ & 0.33 & 1.51 & 0.0 & $3.3(0.5)$ & $-0.16(0.08)$ & $0.01(0.01)$ & $\ldots$ & . \\
\hline 2005jp & 7847 & 2005-11-05.544 & 0.2128 & 1.64 & 32.1 & $-1.3(0.3)$ & $-0.11(0.13)$ & $0.43(0.08)$ & 13. & 3.6 \\
\hline 2006pf & 16567 & $2006-11-23.470$ & 0.3656 & 1.55 & 40.4 & $2.0(0.8)$ & $-0.32(0.10)$ & $0.01(0.02)$ & 12. & 17 \\
\hline $2006 \mathrm{pq}$ & 16618 & $2006-11-23.447$ & 0.193 & 1.48 & 0.0 & $-4.4(0.5)$ & $-0.36(0.13)$ & $0.08(0.07)$ & 4.0 & 51 \\
\hline $2007 \mathrm{my}$ & 19027 & $2007-10-16.244$ & 0.293 & 1.10 & 0.0 & $3.1(0.8)$ & $0.18(0.16)$ & $0.04(0.20)$ & 0.77 & 47 \\
\hline 2007lu & 19029 & $2007-10-14.244$ & 0.319 & 1.13 & 25.5 & $-0.1(1.0)$ & $-0.47(0.07)$ & $0.02(0.03)$ & 2.6 & 63 \\
\hline $2007 \mathrm{ml}$ & 19101 & $2007-10-16.263$ & 0.19 & 1.57 & 0.0 & $-4.0(0.3)$ & $-0.08(0.09)$ & $0.32(0.07)$ & $\cdots$ & $\cdots$ \\
\hline $2007 \mathrm{lw}$ & 19128 & $2007-10-14.267$ & 0.29 & 1.30 & 0.0 & $1.5(0.7)$ & $-0.23(0.09)$ & $0.02(0.04)$ & $\ldots$ & $\ldots$ \\
\hline 2005 jo & 7512 & $2005-11-05.615$ & 0.23 & 1.89 & $\cdots$ & $-1.0(0.4)$ & $-0.27(0.09)$ & $0.26(0.07)$ & $\cdots$ & $\cdots$ \\
\hline $2006 \mathrm{pt}$ & 16644 & 2006-11-23.495 & 0.2990 & 1.49 & $\ldots$ & $-1.9(1.3)$ & $-0.34(0.17)$ & $0.16(0.20)$ & 36. & 8.6 \\
\hline $2006 \mathrm{pz}$ & 16789 & 2006-11-23.525 & 0.3250 & 1.54 & $\ldots$ & $5.6(1.1)$ & $-0.36(0.17)$ & $0.15(0.15)$ & 180 & 1.1 \\
\hline $2007 q u$ & 20829 & $2007-11-12.298$ & 0.31 & 1.14 & $\ldots$ & $-1.2(0.4)$ & $-0.25(0.14)$ & $0.01(0.02)$ & 30. & 2.1 \\
\hline
\end{tabular}

Note. - SNe below the line are those rejected from the Nominal sample. See Section 2.4 for the criteria for inclusion in the Nominal sample.

a Percent galaxy contamination, determined from the synthetic photometry of the spectra in the observer-frame $g$ band.

b Phase relative to the time of $B$-band maximum light.

${ }^{\text {c }} M_{V}(t=0)=-19.504+0.736 \Delta+0.182 \Delta^{2}+5 \log \left(H_{0} / 65 \mathrm{~km} \mathrm{~s}^{-1} \mathrm{Mpc}^{-1}\right) \operatorname{mag}$ (Jha et al. 2007).

d Determined from MLCS fits to our light curves.

e Determined from kcorrect fits to the host-galaxy broad-band photometry.

${ }^{f}$ Percentage of the total star formation over the last $1 \mathrm{Gyr}$ compared to the total star formation over all time, determined from kcorrect fits to the host-galaxy broad-band photometry.

the galaxy SEDs at the position of the SN. The host photometry used to reconstruct the galaxy SEDs is therefore derived from the combination of many Stripe 82 images. Additionally, we employed the SDSS DR6 (Adelman-McCarthy et al. 2008) ugriz photometry for the host galaxies to determine galactic properties such as star-formation rate and mass. Because of the filter gap between the $g$ and $r$ bands, reconstructed SEDs of galaxies in the redshift range $0.27<z<0.33$, in which the $4000 \AA$ break is between the filters, are slightly degenerate. This degeneracy results in many SEDs with similar $g-r$ colors but vastly different $u-g, r-i$, and $r-z$ colors; hence, this method can create unphysical SEDs for these galaxies. However, for the typical galaxy with $z<0.5$, the SEDs are recovered to $\lesssim 0.02 \mathrm{mag}$ in all filters (Blanton et al. 2003; Blanton \& Roweis 2007).

Since there is more variability in the UV SEDs of galaxies than in optical SEDs, the UV region of the SED is more difficult to reconstruct than the optical region. Blanton \& Roweis (2007) tested the predictive properties of kcorrect by determining the SEDs of galaxies with both GALEX and SDSS photometry using only the SDSS photometry. They then compared the residual of the reconstructed GALEX-SDSS colors compared to the observed values. For these galaxies the mean of the residuals was very close to zero. Since we only wish to reconstruct the SED to $\sim 3000 \AA$ in the observer frame, we are confident that the ugriz data are sufficient for reconstructing SEDs over the wavelength region of interest. Additionally, since our SNe are found in Stripe 82, our host photometry is typically much deeper than that of most SDSS galaxies.

Fortuitously, the hosts of SNe 16644 and 16789 have been observed spectroscopically by the SDSS survey (Adelman-McCarthy et al. 2008), allowing a check of the accuracy of this method for reconstructing the galaxy spectra. We also observed five host galaxies with the Magellan Clay telescope after the SN had faded, where we were able to extract a spectrum at the SN position and another spectrum at the position of the host nucleus. Figure 3 compares the observed and reconstructed spectra of the hosts of SNe 7147, 7847, 16567, 16644, and 16789. For the spectra corresponding to the nucleus (including the SDSS spectra), we present the reconstructed spectra of the entire galaxy, and not at the position of the $\mathrm{SN}$. For the spectra corresponding to the SN position, we present the reconstructed spectra at that position.

The data agree very well over most of the wavelengths covered by the observed spectra, suggesting that this procedure is a robust way to determine host-galaxy SEDs. Since SNe 16644 and 16789 are at $z=0.2988$ and 0.3250 , respectively, we can use these observations to understand the potential systematic biases for objects with $0.27<z<0.33$. For most spectra of both the host nucleus and SN position, the observed spectra and reconstructed spectra match very well. However, there are slight differences for SN 16644. The reconstructed spectrum has more near-UV flux at both the nucleus and $\mathrm{SN}$ position. Our sample contains eight $\mathrm{SNe}$ Ia with $0.27<z<0.33$. However, only five of these SNe are used in our final analysis. We have also performed our analysis both with and without these SNe (see Section 2.4), and there is no significant difference in the results.

After generating a reliable host-galaxy SED with the kcorrect routine, we perform two separate methods for determining the SN SED. The methods are different in 

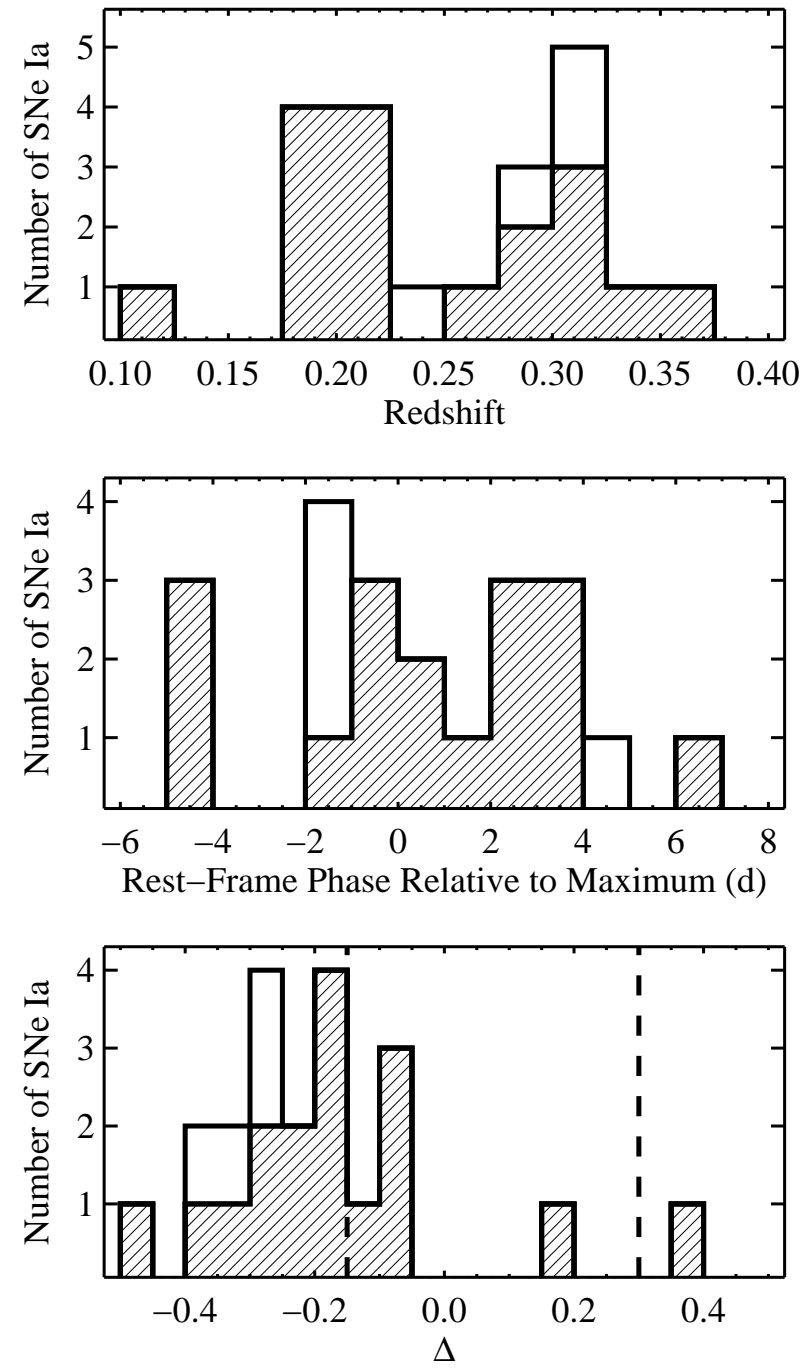

FIG. 2.- Histogram of the redshift (top panel), rest-frame phase as determined from the light curves (middle panel), and $\Delta$ distributions (bottom panel) for the Keck/SDSS SNe Ia presented in this paper. The dashed and open histograms indicate the $\mathrm{SNe}$ in the Nominal sample and additional $\mathrm{SNe}$ in the All sample, respectively; see text (Section 2.4) for a description of these two samples. The dashed lines in the bottom panel mark the regions of high-luminosity $(\Delta<-0.15)$, normal $(-0.15<\Delta<0.3)$, and low-luminosity $(\Delta>0.3) \mathrm{SNe}$, as defined by Jha et al. (2007).

approach, but yield similar results. Both methods are described in detail in Appendix A. Briefly, "photometry matching" is similar to the method employed by Ellis et al. (2008). It requires extracting the SN without attempting to perform local galaxy subtraction and warping the resulting spectrum to the combined host and SN photometry for an aperture corresponding to the combination of slit and seeing. The second method, "color matching," is a novel way to remove galaxy contamination by matching the galaxy-subtracted spectrum to the $\mathrm{SN}$ colors at the time the spectrum was obtained. For our sample, both methods yielded similar results. Both methods have their advantages, but the colormatching method, which removes as much galaxy contamination as possible at the time of extraction, should

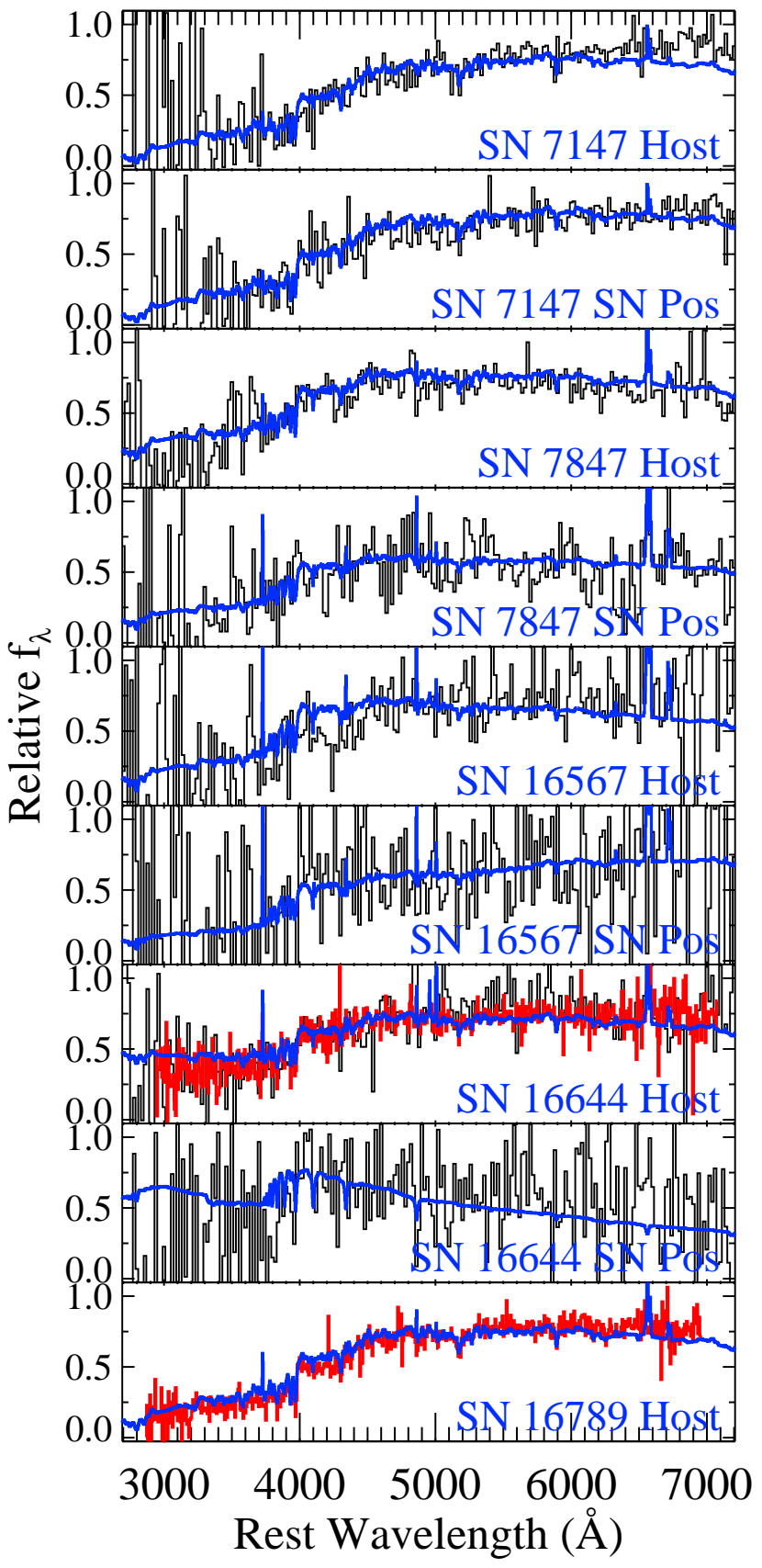

Fig. 3.- Magellan/MagE spectra (black curves) of the nucleus of SDSS J232004.44-000320.1 (the host galaxy of SN 7147; top panel), SDSS J232004.44-000320.1 at the position of SN 7147 (second panel), the nucleus of SDSS J020950.32-000342.0 (the host galaxy of SN 7847; third panel), SDSS J020950.32-000342.0 at the position of SN 7847 (fourth panel), the nucleus of SDSS J014145.08-001121.8 (the host galaxy of SN 16567; fifth panel), SDSS J014145.08-001121.8 at the position of SN 16567 (sixth panel), the nucleus of SDSS J022716.17-002336.5 (the host galaxy of SN 16644; seventh panel), SDSS J022716.17-002336.5 at the position of SN 16644 (eighth panel), and the nucleus of SDSS J025508.24+001405.2 (the host galaxy of SN 16789; bottom panel). The blue curves are the reconstructed galaxy spectra determined by the kcorrect routine. The red curves are SDSS spectra of the galaxies. It is worth noting how well the reconstructed spectra fit the observed spectra over the majority of optical wavelengths. 
have a smaller potential for introducing systematic errors in our sample. We have performed our analysis using both methods, and the results do not change significantly. We will thus focus on the color-matched galaxysubtracted spectra.

\subsection{Composite Spectra}

The SN spectra presented in this paper are generally of very high quality, allowing for studies of individual SNe. However, much additional information can be determined from a composite spectrum of all SNe. We are able to examine both the average properties of the sample as well as small-amplitude spectral features which are below the noise level in individual spectra (Foley et al. 2008a; Ellis et al. 2008; Sullivan et al. 2009).

Using the same procedure to produce the composite spectra as in Folev et al. (2008a), we created several composite spectra from our sample (details of the sample are presented by Foley et al. 2008a and Silverman et al.; submitted)). This includes removing residual emission lines and normalizing the spectra in the region $4500 \leq \lambda \leq 7500 \AA$. The properties of each composite spectrum are listed in Table 2

The various composite spectra are composed of subsamples of our main sample, culled by quality cuts. The "All" spectrum contains the entire sample. All other composite spectra exclude the $\mathrm{SNe}$ which fail the galaxy-contamination correction. The "Nominal" sample includes all $\mathrm{SNe}$ except those that fail the galaxycontamination routine. Since SN Ia spectra evolve with time, having a small time window will decrease discrepancies that are the result of large differences in the epoch of spectra. Accordingly, we created a "Maximum" composite spectrum removing the $\mathrm{SNe}$ with $|t|>3$ days (relative to rest-frame $B$ maximum brightness) from the Nominal sample. To reduce sensitivity to host-galaxy contamination, we created the "Low Galaxy" composite spectrum, removing the $\mathrm{SNe}$ with high galaxy contamination ( $>8 \%$ in the $g$ band) from the Nominal sample. Finally, we created a "Low- $\Delta$ " composite spectrum, removing the low-redshift, high- $\Delta$ SN 7147, which might not be representative of the high-redshift population of SNe Ia and could bias our sample, from the Nominal sample.

We have further considered the effect of having $\mathrm{SNe}$ with $0.27<z<0.33$ in our sample. Although these SNe pass our galaxy-subtraction routines, they may have incorrect galaxy subtraction. We have removed these SNe from the Low-Galaxy sample to create the "No Gap" sample.

To determine how consistent each composite spectrum is with the Nominal composite spectrum in the UV, we have determined the ratio of the UV flux in the wavelength region 2700-4000 $\AA$ for each composite spectrum relative to the Nominal composite spectrum and listed the value in Table 2. The uncertainties given in the table are determined from the $1 \sigma$ boot-strap sampling error spectrum relative to the Nominal composite spectrum. These uncertainty measurements do not include the boot-strap sampling errors of the Nominal spectrum, but we note that the $1 \sigma$ boot-strap sampling error spectrum for the Nominal sample results in a flux that is 0.94 or 1.06 (lower and upper bounds) times the flux of the Nominal composite spectrum in this region. This metric does not contain all pertinent information in the spectrum. For instance, two spectra can have the same average flux over a wavelength range but have significantly discrepant spectral features over that wavelength range. Therefore, we caution use of this number to determine the consistency of two composite spectra, but rather to use these values as indicators of differences while examining the composite spectra (i.e., Figure 4) for a detailed comparison.

Figure 4 shows that to within the uncertainties, the Nominal composite spectrum is similar to all other composite spectra mentioned so far, indicating that the cuts to the Nominal sample do not affect our results, and that the Nominal sample is not significantly biased. Finally, the "Strict" composite spectrum excludes all SNe in at least one of the following categories: fail the galaxycontamination routine, have $|t|>3$ days, have high galaxy contamination, SN 7147, and have $0.27<z<$ 0.33 . The Strict sample consists of just three SNe, and although they do not have any obvious deficiencies, the small sample size means that a single peculiar object may significantly bias the sample.

Examining Figure 4 in detail, we see that the LowGalaxy, No Gap, and Strict composite spectra differ slightly from the Nominal composite spectrum. The differences between the Low-Galaxy and Nominal composite spectra are confined to the regions of the spectrum corresponding to the $\mathrm{Ca} H \& \mathrm{~K}$ feature, and are likely the result of slightly discrepant line velocities or line strengths. The differences in line velocities are almost certainly the result of having slightly different mean phases (e.g., Foley et al. 2011; see also Section 7.1.1). The UV flux for the Low-Galaxy composite spectrum is $1.01{ }_{-0.07}^{+0.07}$, further indicating that the differences are confined to a spectral feature and do not change the continuum. The No Gap and Strict composite spectra have more UV flux than the Nominal composite spectrum (having UV flux of $1.20_{-0.27}^{+0.05}$ and $1.22_{-0.14}^{+0.11}$ times that of the Nominal composite spectrum, respectively). The No Gap spectrum has slightly more UV flux than the Nominal spectrum, but the spectra are consistent within the boot-strap sampling errors. The Strict spectrum has a larger deviation from the Nominal spectrum, but the Strict spectrum consists of only three SN spectra and may be biased. As shown in Section 3 , the Nominal spectrum has more UV flux than the low-redshift composite spectrum; the latter has a UV flux $0.84_{-0.06}^{+0.07}$ times that of the Nominal composite spectrum. The No Gap and Strict spectra have larger deviations with the lowredshift composite spectrum, indicating that quality cuts cannot account for the difference.

We are confident that the Nominal spectrum is an excellent representative of this sample of SNe Ia. No significant differences are observed when restricting our sample to just the SNe near maximum brightness or having low galaxy contamination. There is a minor, but insignificant, difference in the UV continuum of the Nominal and No Gap composite spectra. The No Gap sample contains only six SNe, and is therefore more suscepti- 
TABLE 2

Composite Spectra Information

\begin{tabular}{|c|c|c|c|c|c|c|c|c|c|c|}
\hline $\begin{array}{l}\text { Composite } \\
\text { Name }\end{array}$ & $\begin{array}{l}\text { No. of } \\
\text { Spectra }\end{array}$ & Rejected & $\begin{array}{c}\text { Supernova } \\
\text { High- } \\
\text { Contamination }\end{array}$ & $\begin{array}{l}\text { Included in Subs } \\
0.27<z<0.33\end{array}$ & $\begin{array}{l}\text { imple? } \\
|t|>3 \text { days }\end{array}$ & SN 7147 & $\begin{array}{c}\text { Mean } \\
\text { Phase (days) }\end{array}$ & $\begin{array}{c}\text { Mean } \\
\text { Redshift }\end{array}$ & $\begin{array}{c}\text { Mean } \\
\Delta\end{array}$ & $\begin{array}{l}\text { UV Flux } \\
\text { Relative to } \\
\text { Nominal }\end{array}$ \\
\hline All & 21 & $\mathrm{Y}$ & $\mathrm{Y}$ & $\mathrm{Y}$ & $\mathrm{Y}$ & $\mathrm{Y}$ & -0.4 & 0.23 & -0.15 & $0.99 \stackrel{+0.07}{+0.06}$ \\
\hline Nominal & 17 & $\mathrm{~N}$ & $\mathrm{Y}$ & $\mathrm{Y}$ & $\mathrm{Y}$ & $\mathrm{Y}$ & -0.3 & 0.22 & -0.14 & $\ldots$ \\
\hline Maximum & 10 & $\mathrm{~N}$ & $\mathrm{Y}$ & $\mathrm{Y}$ & $\mathrm{N}$ & $\mathrm{Y}$ & 0.4 & 0.22 & -0.12 & $1.01+0.09$ \\
\hline Low Galaxy & 11 & $\mathrm{~N}$ & $\mathrm{~N}$ & $\mathrm{Y}$ & $\mathrm{Y}$ & $\mathrm{N}$ & 0.0 & 0.23 & -0.16 & $1.01+0.07$ \\
\hline Low $\Delta$ & 16 & $\mathrm{~N}$ & $\mathrm{Y}$ & $\mathrm{Y}$ & $\mathrm{Y}$ & $\mathrm{N}$ & -0.4 & 0.23 & -0.18 & $1.01_{-0.06}^{+0.07}$ \\
\hline No Gap & 6 & $\mathrm{~N}$ & $\mathrm{~N}$ & $\mathrm{~N}$ & $\mathrm{Y}$ & $\mathrm{N}$ & -1.1 & 0.19 & -0.17 & $1.20_{-0.27}^{+0.05}$ \\
\hline Strict & 3 & $\mathrm{~N}$ & $\mathrm{~N}$ & $\mathrm{~N}$ & $\mathrm{~N}$ & $\mathrm{~N}$ & 0.4 & 0.20 & -0.16 & $1.22_{-0.14}^{+0.11}$ \\
\hline \multicolumn{11}{|c|}{ Nominal Subsamples } \\
\hline Active & 7 & $\mathrm{~N}$ & $\mathrm{Y}$ & $\mathrm{Y}$ & $\mathrm{Y}$ & $\mathrm{N}$ & -1.2 & 0.24 & -0.22 & $1.02+0.09$ \\
\hline Passive & 6 & $\mathrm{~N}$ & $\mathrm{Y}$ & $\mathrm{Y}$ & $\mathrm{Y}$ & $\mathrm{Y}$ & 1.0 & 0.19 & -0.03 & $1.00_{-0.15}^{+0.14}$ \\
\hline Low Mass & 10 & $\mathrm{~N}$ & $\mathrm{Y}$ & $\mathrm{Y}$ & $\mathrm{Y}$ & $\mathrm{N}$ & -1.4 & 0.23 & -0.18 & $0.99_{-0.07}^{+0.07}$ \\
\hline High Mass & 5 & $\mathrm{~N}$ & $\mathrm{Y}$ & $\mathrm{Y}$ & $\mathrm{Y}$ & $\mathrm{Y}$ & 0.9 & 0.18 & -0.02 & $1.00_{-0.16}^{+0.16}$ \\
\hline Lower $z$ & 9 & $\mathrm{~N}$ & $\mathrm{Y}$ & $\mathrm{N}$ & $\mathrm{Y}$ & $\mathrm{Y}$ & -1.3 & 0.19 & -0.12 & $1.02+0.09$ \\
\hline Higher $z$ & 8 & $\mathrm{~N}$ & $\mathrm{Y}$ & $\mathrm{Y}$ & $\mathrm{Y}$ & $\mathrm{N}$ & 2.2 & 0.31 & -0.19 & $0.95+0.07$ \\
\hline Negative $\Delta$ & 8 & $\mathrm{~N}$ & $\mathrm{Y}$ & $\mathrm{Y}$ & $\mathrm{Y}$ & $\mathrm{N}$ & -1.2 & 0.24 & -0.28 & $1.07_{-0.10}^{+0.08}$ \\
\hline Zero $\Delta$ & 9 & $\mathrm{~N}$ & $\mathrm{Y}$ & $\mathrm{Y}$ & $\mathrm{Y}$ & $\mathrm{Y}$ & 0.4 & 0.21 & -0.02 & $0.94_{-0.08}^{+0.10}$ \\
\hline
\end{tabular}

ble to biases. Similarly, the Strict composite spectrum, which also deviates slightly from the Nominal composite spectrum at bluer wavelengths, is derived from only three SNe. Given the composite spectra generated from various subsamples and quality-cut tests we have performed, we see no significant biases or systematic errors associated with the Nominal sample and its composite spectrum. Thus, we will use the Nominal spectrum for our analysis.

\section{COMPARISON OF THE KECK/SDSS WITH OTHER SUPERNOVA SAMPLES}

\subsection{Composite Spectra}

In Figure 5, we present the Nominal composite spectrum and compare it with a low-redshift maximum-light composite spectrum. This low-redshift composite spectrum was constructed from the sample of low-redshift spectra used by Foley et al. (2008a), with the additional UV spectra of SN 2005cf (Bufano et al. 2009). This sample represents one of the largest and cleanest sets of low-redshift SN Ia spectra. Most spectra were obtained with the Lick $3 \mathrm{~m}$ telescope and Kast double spectrograph (Miller \& Stone 1993), which provides wellcalibrated spectra from the atmospheric limit to $1 \mu \mathrm{m}$ (see Foley et al. 2008a and Silverman et al., submitted, for additional details). The low-redshift sample used to construct the composite spectrum is of very high quality. The spectra are of well-monitored (both photometrically and spectroscopically) SNe Ia. Each spectrum is selected to have very low galaxy contamination (typically $<3 \%$ ) and very high $\mathrm{S} / \mathrm{N}$ (typically $>50$ ). The phase and $\Delta$ range for the low-redshift sample were chosen to match those of the Keck/SDSS sample. To achieve this, the lowredshift spectra have $-4.8<t<3.0$ days and $\Delta<0.1$. From the fourth and fifth panels of Figure 5, it is clear that the average values for phase and $\Delta$ are very similar between the two samples at all wavelengths.

For wavelengths longer than $\sim 5000 \AA$, the Keck/SDSS and low-redshift composite spectra are remarkably similar. At wavelengths shorter than $\sim 4400 \AA$, however, the spectra differ; the Keck/SDSS spectrum is bluer than its low-redshift counterpart. The difference between the spectra grows larger with decreasing wavelength, becoming more significant by $3000 \AA$. For wavelengths < $3400 \AA$, the Keck/SDSS composite spectrum has $>20 \%$ more flux than the low-redshift composite spectrum. Despite the differences in overall spectral shape, the individual features are all consistent between the two composite spectra.

As mentioned above, it is important to note that the average properties of the spectra (namely redshift, phase, and $\Delta$ ) contributing to a given wavelength bin can change with wavelength. This is particularly the case for the spectra covering slightly different rest-frame wavelength ranges. For the Keck/SDSS sample, the change of average values with wavelength is the result of different redshifts for the SNe, while the average values change in the low-redshift sample mainly because of the different samples for optical and UV spectra.

Considering how well matched the Keck/SDSS and low-redshift composite spectra are in terms of phase and $\Delta$ (the two parameters known to affect an SN spectrum), the differences must come from a different source of variation. Specifically, the samples may be mismatched in some other parameter not known to cause significant color differences, or there is some uncharacterized problem with our data reduction techniques. Below we examine additional parameters and their effects on the spectra.

\subsection{Supernova Colors}

The high quality of our spectra presents the opportunity to derive properties of the individual SNe. It was shown in Section 3.1 that the Keck/SDSS composite spectrum is bluer than the low-redshift composite spectrum at UV wavelengths. Although comparison of the low-redshift composite spectrum to each individual Keck/SDSS SN spectrum is possible, the $\mathrm{S} / \mathrm{N}$ is much lower in the individual spectra. However, by binning the spectra, we may be able to sufficiently increase the $\mathrm{S} / \mathrm{N}$ 


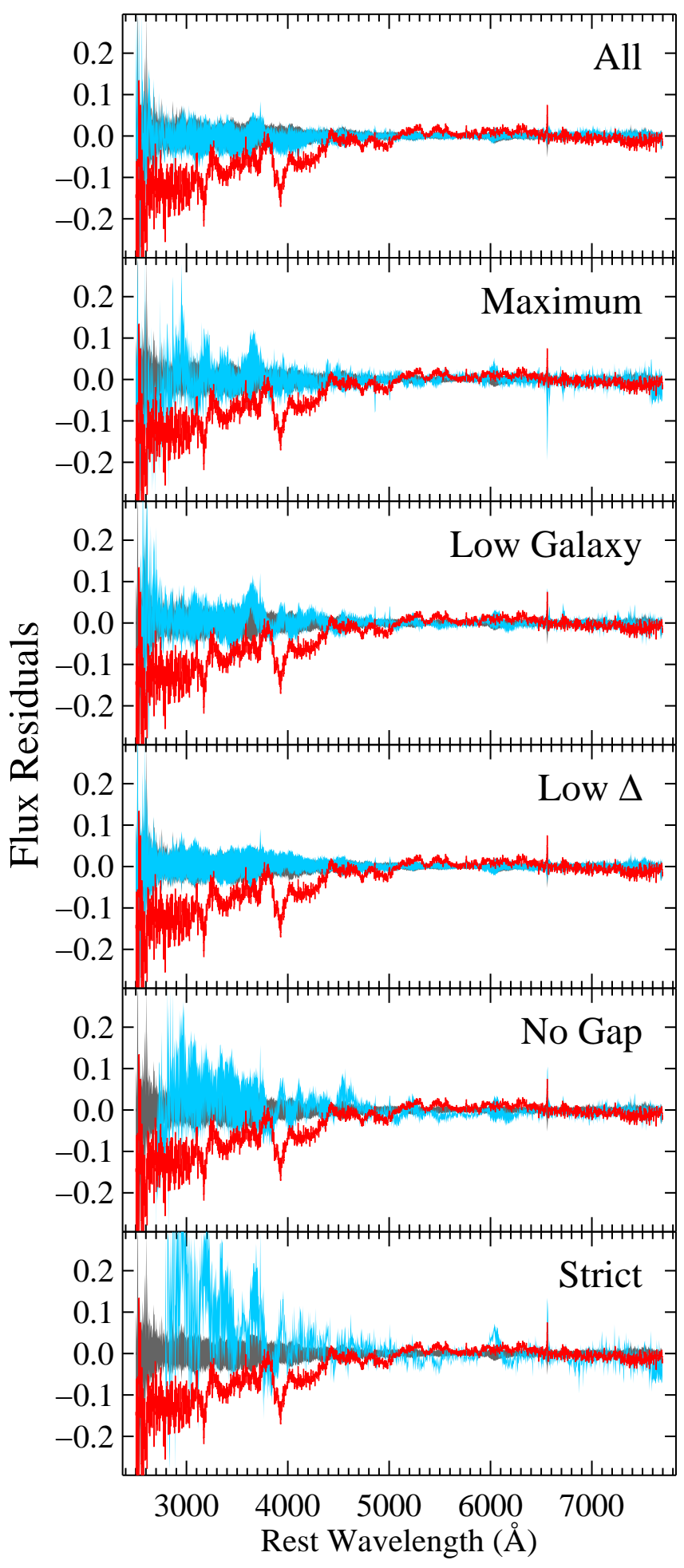

FIG. 4. - The $1 \sigma$ boot-strap sampling error region for the Nominal composite spectrum is displayed in grey. The $1 \sigma$ boot-strap sampling error residuals for the (from top to bottom) All, Maximum, Low Galaxy, Low $\Delta$, No Gap, and Strict composite spectra compared to the Nominal composite spectrum are in light blue (X minus Nominal). The red curve is the residual of the Lick composite and Nominal Keck/SDSS composite spectra (Lick minus Nominal). The grey region and red curves are the same in each panel. The differences between some spectra at $\sim 3650 \AA$ and $\sim 6150 \AA$ are the result of slightly different mean phases, causing a shift in the velocity of the Ca H\&K and Si II $\lambda 6355$ features, respectively (see Section 7.1.1). of the individual spectra to make an interesting measurement.

The binning that we choose is to convolve the spectra with various broad-band filters. Figure 6 displays the Keck/SDSS composite spectrum with the transmission curves of the HST WFC3 F275W and Keck/SDSS ugr filters. We also introduce the "NUV" (near-UV) filter, a top-hat filter that spans the wavelength range 2700$3300 \AA$. The $g$ and $r$ filters probe the area of the spectrum that is mostly consistent between the low-redshift and Keck/SDSS composite spectra; however, the blue edge of the $g$ band does show some differences, so the $g-r$ color of the Keck/SDSS composite spectrum should be bluer than that of the low-redshift composite spectrum. Meanwhile, the F275W, NUV, and $u$ filters probe the region where the spectra diverge.

Using the filter transmission functions, we made color measurements for all of the Keck/SDSS spectra. We have done the same for the low-redshift composite spectrum. Unfortunately, the low-redshift sample rarely has concurrent UV and optical spectra for individual SNe; we therefore are unable to measure the UV minus optical colors of individual SNe. To produce reasonable uncertainties for the Lick composite spectrum, we performed a Monte Carlo analysis of the spectrum using the boot-strap sampling errors to produce several hundred SN spectra. Our measured uncertainties are the standard deviation of the colors for these spectra. The color measurements are presented in Figure 7.

Some of our SNe have rest-frame spectra that do not extend to sufficiently short wavelengths to cover the entire filter. We have taken two approaches to measure synthetic colors for these spectra. First, we set all fluxes for wavelengths shorter than the minimum observed wavelength in the spectrum to zero. For SNe with spectra that extend blueward of $2500 \AA$, where the spectra can be particularly noisy, we also set the flux to zero. Since any flux at these wavelengths will create a bluer color, this measurement sets an upper limit for the color. The second approach sets the fluxes for wavelengths shorter than the minimum observed wavelength in the spectrum to the median flux value for the bluest $100 \AA$ of the spectrum. Since the flux generally increases with wavelength in the UV, this is likely an overestimate of the flux and sets a reasonable lower limit for the color.

As expected from the comparison of the composite spectra, the Keck/SDSS SNe have $g-r$ colors that are similar to those of the low-redshift composite spectrum, with the composite spectra differing by $0.069 \pm 0.025 \mathrm{mag}$. Nearly all of the Keck/SDSS SNe have bluer F275W $-g$ and NUV $-g$ colors than the low-redshift composite spectrum, with the composite spectra differing by 0.618 and $0.294 \mathrm{mag}$ for those colors, respectively. Similarly, most of the Keck/SDSS SNe have bluer $u-g$ colors than the low-redshift composite. Using the Student's $t$ test and assuming that the Lick composite spectrum has the correct colors, we find that the probabilities of the Keck/SDSS and low-redshift samples having the same mean colors are $9.4 \times 10^{-6}, 1.2 \times 10^{-4}$, and $1.5 \times 10^{-2}$ (corresponding to $4.4 \sigma, 3.8 \sigma$, and $2.4 \sigma$ ) for the $\mathrm{F} 275 \mathrm{~W}-g$, $\mathrm{NUV}-g$, and $u-g$ colors, respectively. Conversely, we find that for the $g-r$ color, the two samples are completely consistent with being drawn from the same 


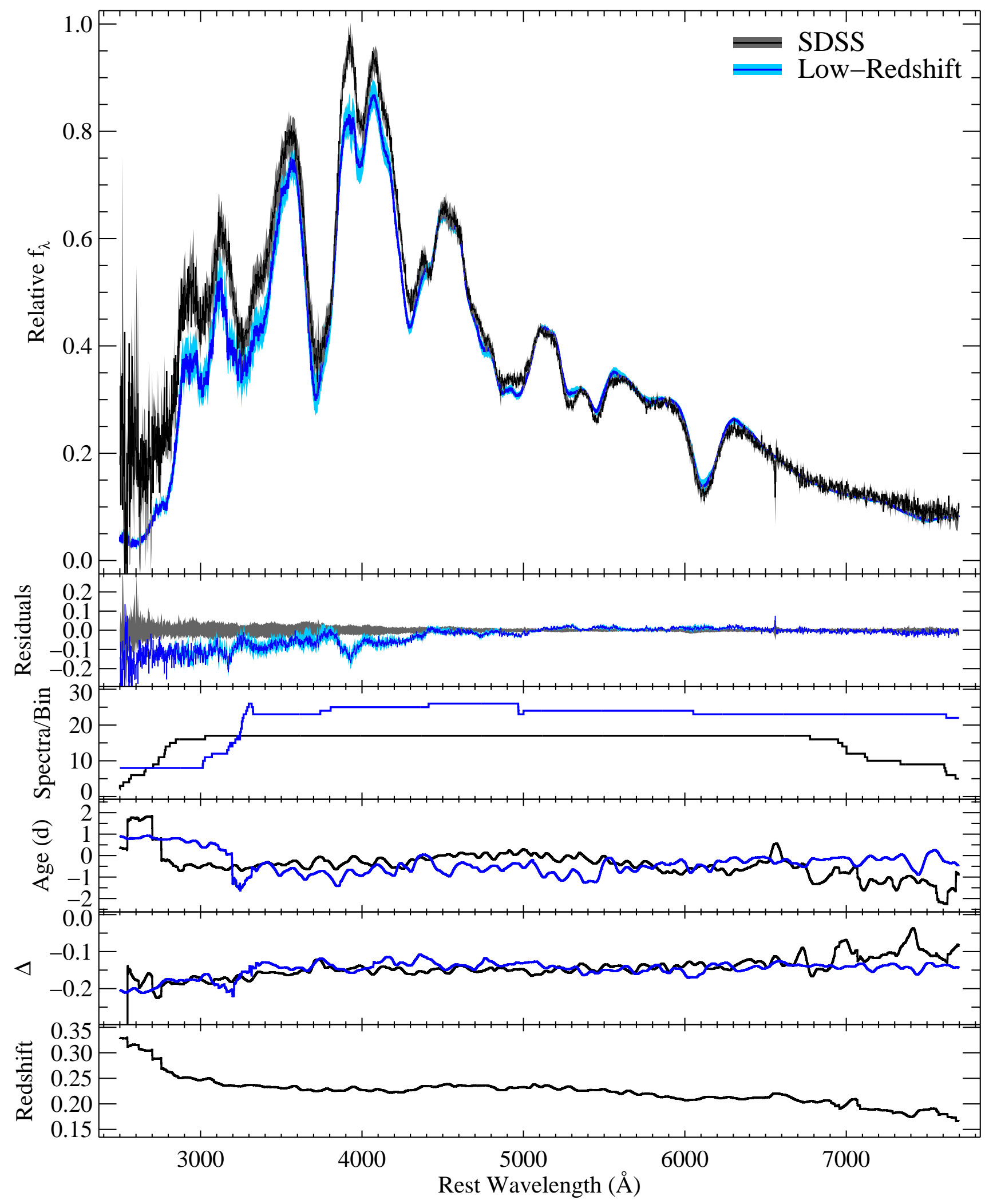

FIG. 5.- (top panel): Composite spectrum created from our Keck/SDSS sample (black curve) compared to the maximum-light lowredshift composite spectrum (blue curve). The spectra are scaled to match in the region $4500 \leq \lambda \leq 7500 \AA$. The grey and light-blue regions are the $1 \sigma$ boot-strap sampling errors for the Keck/SDSS and low-redshift composite spectra, respectively. (second panel): The grey region is the $1 \sigma$ boot-strap sampling region for the Keck/SDSS composite spectrum. The blue curve is the residual of the Keck/SDSS and low-redshift composite spectra. The light-blue region is the residual of the Keck/SDSS composite spectrum and the low-redshift $1 \sigma$ boot-strap sampling region. (third panel): The number of individual spectra contributing to each wavelength bin in the Keck/SDSS (black curve) and low-redshift (blue curve) composite spectra. (fourth panel): The average phase relative to maximum brightness as a function of wavelength for the Keck/SDSS (black curve) and low-redshift (blue curve) composite spectra. (fifth panel): The average value of $\Delta$ as a function of wavelength for the Keck/SDSS (black curve) and low-redshift (blue curve) composite spectra. (bottom panel): The average redshift of the Keck/SDSS composite spectrum as a function of wavelength. 


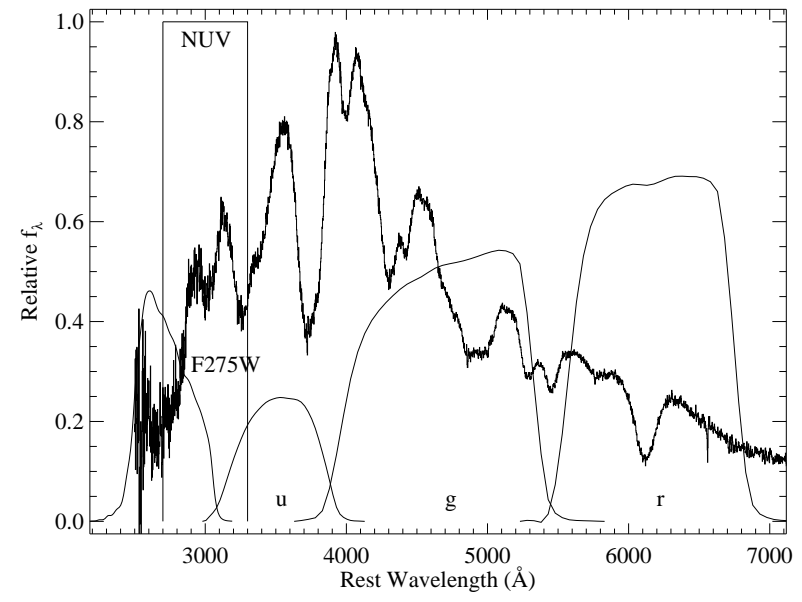

FIG. 6.- The composite Keck/SDSS spectrum shown in Figure!5. Overplotted are (from blue to red) the transmission curves of the HST WFC3 F275W, the newly defined NUV, and the SDSS ugr filters. The noise in the Keck/SDSS composite spectrum for rest wavelengths shorter than $2500 \AA$ is substantial and not plotted.

population.

The color information from the individual spectra shows that the bluer color of the Keck/SDSS composite spectrum is not the result of a minority of SNe heavily influencing the overall spectral shape. The probabilities also indicate that it is unlikely that the low-redshift and Keck/SDSS samples have similar UV colors.

\section{IMPLICATIONS FOR SUPERNOVA COSMOLOGY}

In Section 3, we have shown that the low-redshift and Keck/SDSS samples appear to have different UV SEDs. We will examine possible explanations for these differences, including systematic effects, in Section 7. However, for now, we examine some implications of such a difference for cosmological results. In particular, we explore whether such a mismatch in SEDs could produce the $U$-band anomaly.

As discussed in Section 1, K09 found differences in the rest-frame $U$-band data for low-redshift and highredshift samples that, depending on the treatment of the rest-frame $U$ band, resulted in a systematic shift in the measured value of $w$. This " $U$-band anomaly" is larger for some datasets than others.

We first examine single-band UV distance measurements in Section 4.1, finding that a UV excess produces a distance bias that is in the opposite direction to that of the $U$-band anomaly. In Section 4.2 , we find that differences between the observed and model UV SEDs significantly affect the relationship between light-curve shape and observed color. This explains the relationship between the UV flux deficit found by K09 and the distance modulus offset.

\subsection{Single-Band UV Distance Estimates}

We have shown that there is a mismatch in the UV SEDs of the low-redshift and Keck/SDSS samples of $\mathrm{SNe}$ Ia. In this section, we show that such a mismatch should affect the estimated distance to a SN Ia. However, as we will show in Section 4.2 this effect is dominated by effects related to the correlation between light-curve

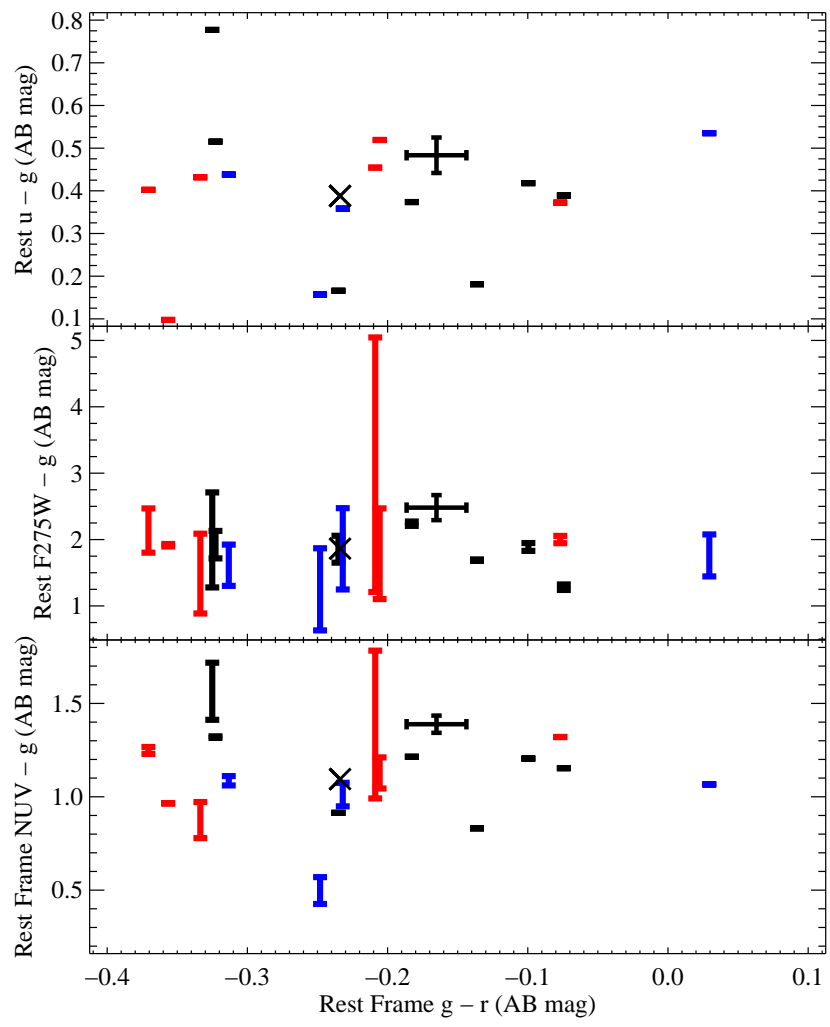

FIG. 7. - Color-color diagrams for the individual Keck/SDSS SNe Ia. The black, blue, and red points correspond to SNe with no, low $(<8 \%)$, and high $(>8 \%)$ host-galaxy contamination, respectively. The black cross with error bars in both directions indicates the colors measured for the low-redshift composite spectrum. The black "X" indicates the Keck/SDSS composite spectrum. The color measurements are derived by convolving the individual Keck/SDSS SN spectra with filter transmission curves. Since not all of the spectra cover the entire wavelength range of each filter, there is a range of color for some points. The upper limits are derived by setting all flux for wavelengths shorter than the shortest observed wavelength of a spectrum to zero. The lower limits are derived by setting all flux for wavelengths shorter than the shortest observed wavelength of a spectrum to the median flux value of the bluest $100 \AA$ of the spectrum.

shape and color when using multiple-band data, and is likely not the cause of the $U$-band anomaly, but could be important for some high-redshift SNe Ia, which have limited color information.

Some light-curve fitters train on a low-redshift sample and apply the measured relationships between lightcurve shape and color from that sample to high-redshift SNe; MLCS2k2 (Jha et al. 2007), in particular, uses this methodology. Other light-curve fitters, such as SALT2 (Guy et al. 2007), determine correlations between lightcurve shape, color, and peak luminosity using all SNe in its sample, including both low and high-redshift data.

MLCS2k2 is trained on the low-redshift sample of $\mathrm{SNe}$ Ia. If the average SEDs of low and high-redshift $\mathrm{SNe}$ Ia in our observed samples are properly described by the spectra presented in Section 3 , then MLCS2k2 will underpredict the flux of a high-redshift SN Ia in the rest-frame UV.

Imagine that we observed an SN Ia at high redshift in only rest-frame $U$. The MLCS2k2 estimate of $M_{U \text {,true }}$, 
the real peak absolute magnitude of the $\mathrm{SN}$ in $U$, will be overestimated (MLCS2k2 will underestimate the luminosity of the $\mathrm{SN}$ in $U$ ). We can define this difference as

$$
M_{U, \text { true }}=M_{U, \mathrm{MLCS}}-\Delta M,
$$

where $M_{U, \mathrm{MLCS}}$ is the MLCS2k2 estimate of the true value of $M_{U \text {,true }}$, and $\Delta M$ is the magnitude difference between the estimate and the true value. With our assumption and formalism, $\Delta M \geq 0 \mathrm{mag}$.

Since the observed magnitude, $U$, does not rely on any assumptions about the SN SED, we find

$$
\begin{aligned}
\mu_{\text {true }} & =U-M_{U, \text { true }}=U-M_{U, \mathrm{MLCS}}+\Delta M \\
& =\mu_{\mathrm{MLCS}}+\Delta M,
\end{aligned}
$$

where $\mu_{\text {true }}$ and $\mu_{\text {MLCS }}$ are the true and MLCS2k2measured distance moduli, respectively. Since $\Delta M \geq$ $0 \mathrm{mag}, \mathrm{MLCS} 2 \mathrm{k} 2$ is underestimating the distance to this $\mathrm{SN}$.

K09 found that MLCS2k2 fits including and excluding the rest-frame $U$ band produced a difference in average distance moduli for the SDSS-II sample of $\mu_{\mathrm{no} U}-\mu \simeq$ $0.1-0.15 \mathrm{mag}$, where $\mu_{\mathrm{no} U}$ and $\mu$ are the distance moduli measured with MLCS2k2 excluding and including the rest-frame $U$ band, respectively. If we assume that ignoring the rest-frame $U$ band will avoid the complications of any potential SED differences in the UV, then $\mu_{\text {no } U}$ should be an unbiased measurement of the true distance modulus, while $\mu$ represents the measurement biased by the rest-frame $U$-band data. Under this assumption, $\mu_{\mathrm{no} U}=\mu_{\text {true }}$ and $\mu=\mu_{\mathrm{MLCS}}$. Then we have

$$
\begin{aligned}
\Delta M & =\mu_{\mathrm{true}}-\mu_{\mathrm{MLCS}} \\
& =\mu_{\mathrm{no} U}-\mu \\
& \simeq 0.1-0.15 \mathrm{mag} .
\end{aligned}
$$

Since $\Delta M>0 \mathrm{mag}$, the effect of the $U$-band anomaly is in the same direction as that expected by an excess of UV flux in high-redshift SNe Ia relative to low-redshift SNe Ia.

To determine if the amplitude of the effect is the same, we can simply examine the $u-r$ color of the Keck/SDSS and low-redshift composite spectra. For this, we use the fact that the SEDs of both composite spectra are very similar in the $r$ band; therefore, most differences in this color will be from differences in the $u$ band. In Section 3.2. we showed that the difference in $u-r$ colors of the composite spectra is $\Delta(u-r)=0.15 \pm 0.05 \mathrm{mag}$. This difference is also of the same magnitude as seen by K09.

Another way in which a difference in UV SEDs can affect the measured distance is the treatment of dust. Since MLCS2k2 uses the SN colors to estimate the hostgalaxy extinction, a color difference not accounted for by the model will result in an incorrect measurement of this extinction. (SALT2 does not attempt to directly measure extinction, but the measured distance is still affected by $\mathrm{SN}$ color in a similar manner.)

MLCS2k2 models all SNe Ia with an intrinsic color (for our purposes, we will use rest-frame $u-r$ as an example) that depends on light-curve shape and has an intrinsic scatter. If the measured color is redder than the expected color for a given light-curve shape, then MLCS2k2 models the difference in color as host-galaxy reddening. If high-redshift SNe Ia have bluer $u-r$ colors than low-redshift SNe Ia (and our measurements of the composite spectra support this), then MLCS2k2 will not properly measure the host-galaxy reddening, estimating a value that is less than the true value. Another way to interpret this is that the MLCS2k2 zero-reddening color, $(u-r)_{0, \text { MLCS }}$, is too red.

We can describe this effect mathematically. First, we define the real extinction in the $V$ band as

$$
A_{V, \text { true }}=\alpha\left[(u-r)-(u-r)_{0, \text { true }}\right],
$$

where $\alpha$ is a factor that converts the color difference from the zero-reddening value to extinction in the $V$ band (and is in part related to $\left.R_{V}\right),(u-r)$ is the measured color, and $(u-r)_{0, \text { true }}$ is the real zero-reddening color. The above effect can be characterized as

$$
\begin{aligned}
A_{V, \mathrm{MLCS}} & =\alpha\left[(u-r)-(u-r)_{0, \mathrm{MLCS}}\right] \\
& =\alpha\left[(u-r)-\left((u-r)_{0, \text { true }}+\Delta(u-r)\right)\right] \\
& =A_{V, \text { true }}-\alpha[\Delta(u-r)],
\end{aligned}
$$

where $\Delta(u-r)$ is the difference between the MLCS2k2 and real zero-reddening color. Since the real zeroreddening color is bluer than the MLCS2k2 zeroreddening color, $\Delta(u-r) \geq 0$ mag and $A_{V, \text { MLCS }} \leq$ $A_{V \text {,true. }}$

Under these simple assumptions, we expect MLCS2k2 to underestimate the extinction to a given SN Ia, and therefore to underestimate its luminosity and distance. Since this effect is in the same direction as the luminosity effect described above, the two effects should not cancel each other out.

Although our simple analysis shows that a difference in the UV SED for low and high-redshift SNe Ia, similar to what is seen between the low-redshift and Keck/SDSS samples, can produce a difference in the inferred distances (both in amplitude and direction) as the $U$-band anomaly, there is a significant observational problem: the SDSS-II sample of K09 has, on average, lower $U$ band flux than their adopted low-redshift sample. This is opposite to the difference seen in Figure 5] We discuss the potential reasons for the difference between the Keck/SDSS and overall SDSS-II samples in Section 7.1.1. Below, we discuss how the full SDSS-II sample has the opposite UV flux difference (relative to the low-redshift sample) yet affects the distance modulus in the same direction as shown for the single-band case.

\subsection{Multi-Band UV Distance Estimates}

Our presentation of light-curve fitters in the previous section is extremely simplified. In reality, all light-curve data, including bands other than rest-frame $U$ and data obtained away from maximum light, affect the final estimated distance. Although the difference in the SEDs results in a distance modulus difference that is of the same magnitude of the $U$-band anomaly, K09 calculated distance moduli using three filters, and naively, one would expect the rest-frame $U$ band to contribute about one third of any difference.

Additionally, colors, which are only measured with multi-band data, can influence the inferred distance modulus through the correlations between observed color, light-curve shape, and peak luminosity. In the MLCS 
framework, an SN that is bluer than the SN model will be assigned a $\Delta$ (the MLCS2k2 light-curve shape parameter) that is smaller than it should, and the SN is assumed to be intrinsically brighter than it is. That is,

$$
M_{\text {true }}>M_{\text {MLCS }} \text {. }
$$

From this, we see that the distance modulus is affected such that

$$
\begin{aligned}
\mu_{\mathrm{true}} & =m-M_{\mathrm{true}} \\
& <m-M_{\mathrm{MLCS}} \\
& =\mu_{\mathrm{MLCS}} .
\end{aligned}
$$

Therefore,

$$
\Delta M=\mu_{\text {true }}-\mu_{\mathrm{MLCS}}<0 \mathrm{mag},
$$

which is opposite of the effects noted above.

To assess the impact of these various effects on the inferred distance, we performed multiple simulations using the SNANA framework (Kessler et al. 2009b). We simulated a large sample of $U B V$ light curves for SNe Ia at $z=0.01$ with $A_{V}=0 \mathrm{mag}$ to reduce the affects of $K$-corrections and extinction.

We fit the light curves in a variety of ways with MLCS2k2. First, we fit only the $U$-band data. As expected (Section 4.1), we find $\Delta M=\mu_{\text {true }}-\mu_{\mathrm{MLCS}}>$ 0 mag. Next, we fit the full $U B V$ and only the $B V$ light curves with $A_{V}=0 \mathrm{mag}$ for all SNe. This isolates any potential correlations between color-luminosity and color-extinction relationships. We find

$$
\mu_{B V}-\mu_{U B V}=-0.11 \mathrm{mag},
$$

which is opposite to the effect described in Section 4.1 Clearly the multi-band data are important. If we fix the $U$-band $K$-correction, $K_{U U}$, to zero, the results hold to within $0.01 \mathrm{mag}$; therefore, $K$-corrections are not a dominant factor.

Next, we fit the light curves fixing the light-curve shape parameter, $\Delta$, for each SN to be the same as the simulated value for that SN. In this case, we find

$$
\mu_{B V}-\mu_{U B V}=+0.05 \mathrm{mag},
$$

in rough agreement with the results of Section 4.1. We have performed a similar analysis with an SDSS-like simulation $(0.1<z<0.3$ and gri filters) and obtained similar results.

From these findings, we can conclude that including miscalibrated $U$-band data in the light-curve fits dramatically affects the relation between light-curve shape and observed color, which in turn affects the measured distance modulus. The Keck/SDSS sample has less UV flux than the low-redshift sample, on average, and therefore has $\Delta M>0 \mathrm{mag}$.

\section{SUBSAMPLES OF THE KECK/SDSS SAMPLE}

In Section 2.4 we created several subsamples of the entire set of Keck/SDSS SNe to test if various quality cuts affected the composite spectra. We determined through this process that we should exclude SNe where the galaxy subtraction procedure failed.

Since our sample of high-redshift SNe Ia is relatively large, we are also able to create subsamples based on physical parameters. As we discussed in Section 3.1. the

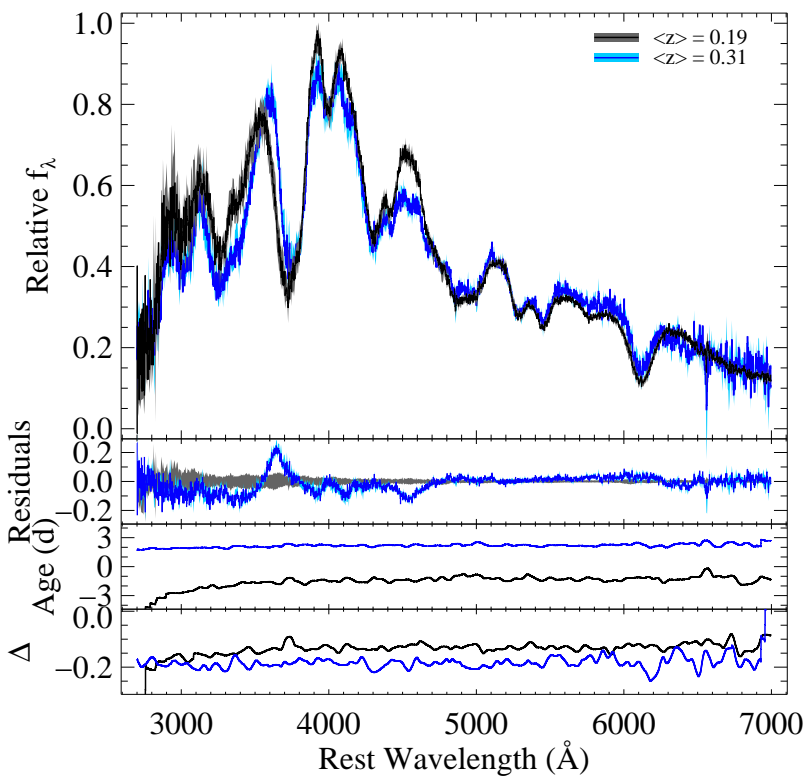

FIG. 8.- (top panel): Composite spectrum created from Keck/SDSS SNe with $z<0.25$ (black curve) and $z>0.25$ (blue curve). The grey and light-blue regions are the $1 \sigma$ boot-strap sampling errors for the lower-redshift and higher-redshift composite spectra, respectively. (second panel): The grey region is the $1 \sigma$ boot-strap sampling region for the lower-redshift composite spectrum. The blue curve is the residual of the lower-redshift and higher-redshift composite spectra. The light-blue region is the residual of the lower-redshift composite spectrum and the higherredshift $1 \sigma$ boot-strap sampling region. (third panel): The average phase relative to maximum brightness as a function of wavelength for the lower-redshift (black curve) and higher-redshift (blue curve) composite spectra. (bottom panel): The average value of $\Delta$ as a function of wavelength for the lower-redshift (black curve) and higher-redshift (blue curve) composite spectra. The UV flux is similar in both spectra.

Nominal Keck/SDSS and low-redshift composite spectra have very similar average phases and light-curve shapes, the dominant factors in spectral differences. Below we will separate the Nominal sample based on other physical properties to investigate possible physical reasons for the difference in UV SED between the Keck/SDSS and lowredshift samples. Some properties of the subsamples are listed in Table 2 .

\subsection{Redshift}

Splitting the Nominal sample by redshift (using the mean redshift of the Nominal sample, 0.25, as the division), we are able to create two samples: a lower-redshift sample of nine $\mathrm{SNe}$ with $\langle z\rangle=0.19,\langle t\rangle=-1.3$ days, and $\langle\Delta\rangle=-0.12$, and a higher-redshift sample of eight SNe with $\langle z\rangle=0.31,\langle t\rangle=2.2$ days, and $\langle\Delta\rangle=-0.19$. Composite spectra created from these samples are shown in Figure 8 .

The composite spectra from the lower-redshift and higher-redshift subsamples are very similar at most wavelengths. The major difference is in the blue wing of the $\mathrm{Ca} H \& \mathrm{~K}$ feature, which extends farther to shorter wavelengths in the lower-redshift composite spectrum. As the velocity of the minimum of the $\mathrm{Ca} H \& \mathrm{~K}$ feature has a range of about $-12,000$ to $-22,000 \mathrm{~km} \mathrm{~s}^{-1}$ at $t=-5$ days and about $-11,000$ to $-18,000 \mathrm{~km} \mathrm{~s}^{-1}$ at $t=5$ days, with the line wider at earlier times (e.g., Folev et al. 2011; see also Section [7.1.1), the difference 
in the two composite spectra is likely to be an age effect; the lower-redshift sample has an average phase 2.8 days earlier than the average phase of the higher-redshift sample.

The other difference between the spectra is at $\sim 4500 \AA$, a peak between absorption features. Although it may be attributed to the different average redshifts, it is more likely that this difference is also an age effect.

The lower-redshift composite spectrum appears to have slightly more UV flux than the higher-redshift composite spectrum, but the continua are still consistent within the boot-strap sampling errors. Since the SDSS-II sample should have relatively little Malmquist-associated biases at $z \approx 0.2$, the lack of a difference in UV SED between the two composite spectra indicates that these sorts of biases are not creating the mismatch in SEDs between the low-redshift and Keck/SDSS samples.

\subsection{Host-Galaxy Star Formation and Mass}

Recently, there have been some indications that SNe Ia from different host environments have different peak luminosities after correction for light-curve shape and color (Hicken et al. 2009b; Kelly et al. 2010; Sullivan et al. 2010; Lampeitl et al. 2010b). Although these studies have noticed a correlation between Hubble residuals and both morphology (Hicken et al. 2009b) and mass or starformation rate (Kelly et al. 2010; Sullivan et al. 2010; Lampeitl et al. 2010b), the cause of the correlation is unclear, and it is not known whether the correlation is physical or related to the light-curve fitting.

As a byproduct of fitting galaxy SEDs to the hostgalaxy photometry, we can obtain a measurement of the mass and star-formation history of the host galaxies. The kcorrect routine constructs galaxy SEDs from several hundred spectral templates. Using the relative importance of each template in the galaxy SED, the star-formation history of the galaxy is reconstructed (Blanton \& Roweis 2007). Once we determine the luminosity of the galaxy (using the photometry, redshifts, and a cosmological model), the spectral templates provide a measurement of the stellar mass of the galaxy. Although kcorrect does not directly output an uncertainty for its mass estimate, the typical uncertainty is 0.1 dex (Blanton \& Roweis 2007).

From the Nominal sample, there are four SNe with no host detected in the SDSS images. Consequently, we have no information about the star-formation rate of these galaxies (although they likely have high specific star-formation rates). We can, with reasonable confidence, say that the undetected host galaxies are of relatively low mass. As an example, the host of SN 16567 at $z=0.37$ (the highest redshift galaxy in the sample) has a host galaxy with $M=11.8 \times 10^{9} \mathrm{M}_{\odot}$. Undetected galaxies at lower redshift $(z=0.19,0.215,0.29$, and 0.33$)$ almost certainly have lower mass.

With the full Nominal sample and the subsample of 13 SNe Ia having host-galaxy detections, we are able to respectively split the $\mathrm{SNe}$ by total stellar mass and $B 1000$, the percentage of the total star formation over the previous 1 Gyr compared to the total star formation over all time. This information is listed in Table 11. The full sample can easily be divided at $M=5 \times 10^{9} \mathrm{M}_{\odot}$ into two groups. We include SNe $6933(z=0.215)$ and

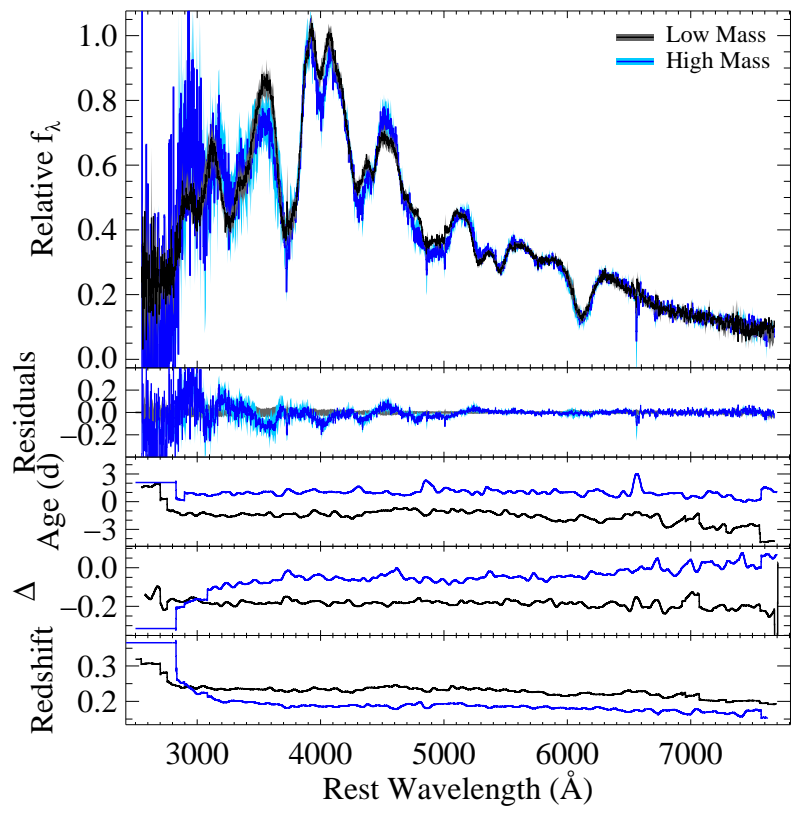

FIG. 9.- (top panel): Composite spectra from Keck/SDSS SNe with host galaxies that have $M<5 \times 10^{9} \mathrm{M}_{\odot}$ (black curve) and $M>5 \times 10^{9} \mathrm{M}_{\odot}$ (blue curve). The grey and light-blue regions are the $1 \sigma$ boot-strap sampling errors for the low-mass and highmass composite spectra, respectively. (second panel): The grey region is the $1 \sigma$ boot-strap sampling region for the low-mass composite spectrum. The blue curve is the residual of the low-mass and high-mass composite spectra. The light-blue region is the residual of the low-mass composite spectrum and the high-mass $1 \sigma$ bootstrap sampling region. (fourth panel): The average phase relative to maximum brightness as a function of wavelength for the lowmass (black curve) and high-mass (blue curve) composite spectra. (fifth panel): The average value of $\Delta$ as a function of wavelength for the low-mass (black curve) and high-mass (blue curve) composite spectra. (bottom panel): The average redshift of the low-mass composite spectrum as a function of wavelength. The two spectra are relatively similar, but there is perhaps a slight difference in the UV slope, with the high-mass composite spectrum having a flatter spectrum from $2900-3500 \AA$.

$19101(z=0.19)$, which do not have detected hosts, in the low-mass category. Since SNe $7475(z=0.33)$ and $19128(z=0.29)$ could conceivably have a mass near our chosen division, we do not include either SN in either subsample, resulting in a final sample of $15 \mathrm{SNe}$ from which we examine the SED dependence on host-galaxy mass. The subsample of SNe with B1000 measurements is divided into two groups, "active" and "passive" having $B 1000>46$ and $B 1000<17$, respectively.

Not surprisingly, most passive hosts have a large mass and most active hosts have a small mass. Because of the larger number of SNe in the mass segregated sample, we study that one in more detail. We note that the same trends appear when using the passive/active subsamples. We present the composite spectra created from the lowmass and high-mass subsamples in Figure 9.

The low-mass and high-mass composite spectra have average values of $\langle z\rangle=0.23,\langle t\rangle=-1.4$ days, $\langle\Delta\rangle=$ -0.18 , and $\langle z\rangle=0.18,\langle t\rangle=0.9$ days, $\langle\Delta\rangle=-0.02$, respectively. Although the composite spectra from the low-mass and high-mass subsamples (which are essentially the same as the active and passive subsamples, respectively) are very similar in the optical, there are some minor differences in the UV. In particular, the peak at 
$\sim 3500 \AA$ is higher in the low-mass composite spectrum, while the peak at $\sim 2900 \AA$ is lower in the low-mass composite spectrum. This results in a relatively flat spectral slope for the high-mass composite spectrum in the range 2900-3500 A. The composite spectra do differ by $\sim 2$ days in phase and by $\sim 0.15$ in $\Delta$; however, for the low-redshift sample, Folev et al. (2008a) did not see the "steepness" of the near-UV change as a function of mean phase or $\Delta$ (on the other hand, FFJ did detect a strong correlation between $\Delta$ and the slope of the SN's spectrum between 2770 and $2900 \AA$ ). We therefore believe that it is unlikely that the discrepancies in the mean phase or $\Delta$ have caused the differences in these composite spectra. Unfortunately, the small sample sizes makes these differences marginally significant, and we require more SNe to determine whether this trend continues.

Despite the differences in these composite spectra, both have a UV excess compared to the low-redshift composite spectrum. It is therefore unlikely that the UV excess is the result of a biased host population.

\subsection{Light-Curve Shape}

Splitting the Nominal sample by $\Delta$ (using the median value of the Nominal sample, $\Delta=-0.169$, as the division), we are able to create two subsamples, one with a smaller average $\Delta$ (the "Negative $\Delta$ ") with eight $\mathrm{SNe}$ and average values of $\langle z\rangle=0.24,\langle t\rangle=-1.2$ days, and $\langle\Delta\rangle=-0.28$, and one with a larger average $\Delta$ (the "Zero $\Delta ")$ with nine $\mathrm{SNe}$ and average values of $\langle z\rangle=0.21$, $\langle t\rangle=0.4$ days, and $\langle\Delta\rangle=-0.02$. Composite spectra created from these samples are shown in Figure 10.

The composite spectra from the zero and negative- $\Delta$ subsamples exhibit many differences at all wavelengths. The Si II $\lambda 5972$ feature is weak in the negative- $\Delta$ composite spectrum. The Si II $\lambda 6355$ feature is also significantly weaker in the negative- $\Delta$ composite spectrum compared to the zero- $\Delta$ composite spectrum. There are also many insignificant differences redward of $\sim 4300 \AA$. Clearly the optical SEDs of SNe in these subsamples are different, but the individual features are similar enough that the spectra are consistent within the boot-strap sampling errors.

The spectra continue to deviate at bluer wavelengths. In addition to weak Si II $\lambda 5972$ and $\mathrm{Si}$ II $\lambda 6355$ features, the negative- $\Delta$ composite spectrum has a relatively weak Si II $\lambda 4130$ line. The Ca H\&K feature is also weaker in the negative- $\Delta$ spectrum. The blue edge of the $\mathrm{Ca} \mathrm{H} \& \mathrm{~K}$ feature has higher flux in the negative$\Delta$ spectrum, indicating that the apparent weakness of Ca H\&K could be caused by more continuum flux at the position of the feature; however, scaling the spectra to that peak, the Ca H\&K feature is still weaker in the negative- $\Delta$ spectrum. Therefore, we believe that this difference is inherent to the absorption feature. These differences can be described by the $\mathcal{R}(\mathrm{Ca}$ II) parameter of Nugent et al. (1995), which is the ratio of the flux at the red edge of the Ca H\&K feature to that of the flux at the blue edge. The Keck/SDSS composite spectra follow the trend of more luminous (or SNe with smaller $\Delta$ ) corresponding to smaller $\mathcal{R}(\mathrm{Ca}$ II $)$. These effects have also been seen when comparing low-redshift composite spectra (Foley et al. 2008a).

The UV flux is clearly higher in the negative- $\Delta$ spec-

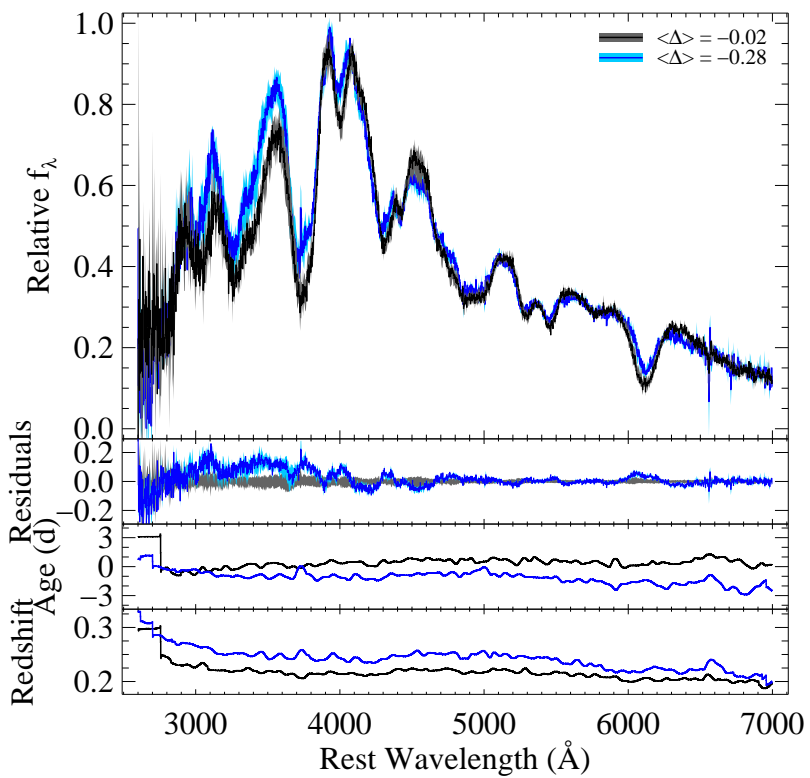

Fig. 10.-(top panel): Composite spectra from Keck/SDSS SNe with $\Delta \geq-0.169$ (black curve) and $\Delta<-0.169$ (blue curve). The grey and light-blue regions are the $1 \sigma$ boot-strap sampling errors for the negative- $\Delta$ and zero- $\Delta$ composite spectra, respectively. (second panel): The grey region is the $1 \sigma$ boot-strap sampling region for the negative- $\Delta$ composite spectrum. The blue curve is the residual of the negative- $\Delta$ and zero- $\Delta$ composite spectra. The light-blue region is the residual of the negative- $\Delta$ composite spectrum and the zero- $\Delta 1 \sigma$ boot-strap sampling region. (fourth panel): The average phase relative to maximum brightness as a function of wavelength for the negative- $\Delta$ (black curve) and zero- $\Delta$ (blue curve) composite spectra. (bottom panel): The average redshift of the negative- $\Delta$ composite spectrum as a function of wavelength. Both spectra have similar UV flux.

trum in the range $3000-3600 \AA$. The absorption minima of the $\mathrm{Fe}$ II $\lambda 3250$ feature is at $-23,500$ and $-21,300 \mathrm{~km} \mathrm{~s}^{-1}$ for the negative and zero- $\Delta$ spectra, respectively. This is consistent with the rough trend that low- $\Delta$ SNe have a higher velocity for this feature at maximum brightness (FFJ). The peaks just blueward and redward of this feature are called $\lambda_{1}$ and $\lambda_{2}$, respectively, by Ellis et al. (2008). The negative- $\Delta$ composite spectrum has a smaller wavelength for the peak of $\lambda_{2}$ than the zero- $\Delta$ composite spectrum. Ellis et al. (2008) found that there was significant scatter in the position of these features and that the position was strongly correlated with phase.

Examining Figure 15 of Ellis et al. (2008), we see that the Keck/SDSS sample shows similar results when separating the sample into two bins based on light-curve shape. Their "high-stretch" (corresponding to smaller $\Delta$ ) spectrum has stronger UV flux, a smaller $\mathcal{R}(\mathrm{Ca}$ II), a higher velocity for the Fe II $\lambda 3250$ feature, and a shorter peak wavelength for $\lambda_{2}$. All of these characteristics are also seen in the Keck/SDSS sample.

\section{INVESTIGATION OF THE UV RATIO}

The set of Keck/SDSS SN Ia spectra is comparable to the current low-redshift UV sample in size and quality (FFJ). The low-redshift UV sample was mostly obtained with the International Ultraviolet Explorer (IUE) telescope, including almost all spectra near maximum light (SN 2005cf was observed by Swift; Bufano et al. 2009). There are 15 low-redshift SN Ia UV spectra with 


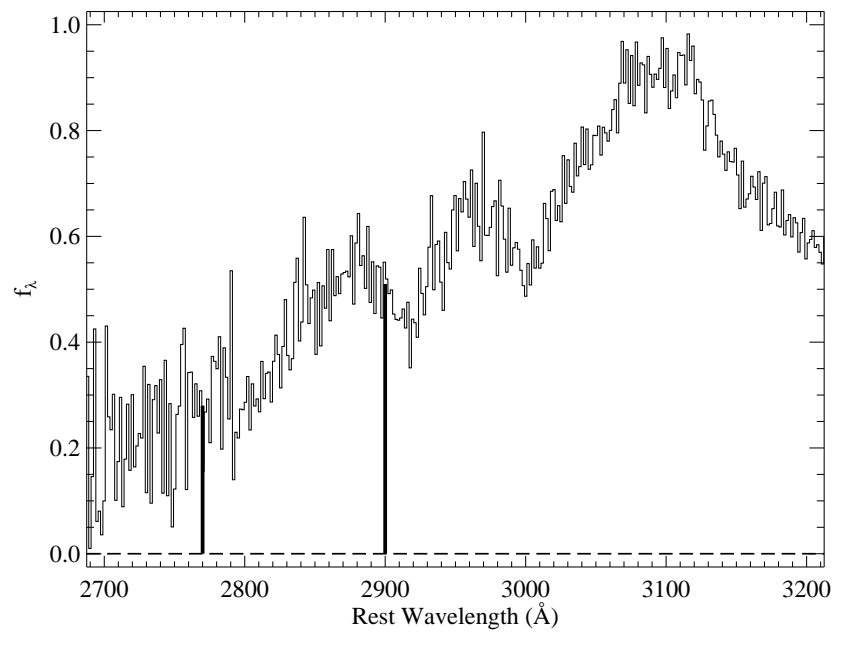

FIG. 11. - Rest-frame UV spectrum of SN $16618(z=0.193$; $t=-4.4$ days. Marked are the wavelengths (2770 and $2900 \AA)$ and corresponding fluxes used for calculating the UV ratio, $\mathcal{R}_{U V}$.

$-3<t<3$ days from 7 SNe Ia (SNe 1980N, 1981B 1986G, 1990N, 1991T, 1992A, 2005cf). These "classic" SNe Ia all have precise distance measurements from independent Cepheid observations or surface brightness fluctuations. Consequently, FFJ were able to determine a relationship between the ratio of the flux of the SN spectrum at 2770 and $2900 \AA, \mathcal{R}_{U V}$ (the "UV ratio"), and peak luminosity. An example of how the UV ratio is calculated is shown in Figure 11.

The UV ratio is an excellent luminosity indicator for these six low-redshift SNe Ia. Using a linear relationship, we see that the UV ratio yields a scatter in the peak absolute magnitudes of the $\mathrm{SNe}$ of $0.21 \mathrm{mag}$, comparable to the scatter found when using the luminosity vs. lightcurve shape relationship ( $\sigma \approx 0.17$ for this sample; FFJ). Furthermore, combining the UV ratio and the luminosity vs. light-curve shape relationship, FFJ were able to reduce the scatter to $0.09 \mathrm{mag}$.

For a subsample of our Keck/SDSS SNe, we possess accurately fluxed-calibrated UV spectra (based on our galaxy-subtraction techniques) that have reasonable $\mathrm{S} / \mathrm{N}$ at $2770 \AA$ to measure $\mathcal{R}_{U V}$. These $\mathrm{SNe}$ are all SNe from the Nominal sample observed in 2006 and 2007, supplemented by those with $z \geq 0.215$ from 2005 . In Figure 12 we present the measured UV ratios from our sample as a function of $\Delta$ and compare to the low-redshift values. The SDSS-II light curves were fit with a slightly different version of MLCS2k2 than that of the low-redshift sample (see Section 2.2). Fitting a subset of low-redshift $\mathrm{SNe}$ with both versions of MLCS2k2, we see an offset of 0.01 and a root-mean-square scatter of 0.02 in $\Delta$ between the two outputs. The larger uncertainty in $\Delta$ for the Keck/SDSS SNe is the result of slightly lower quality (both $\mathrm{S} / \mathrm{N}$ and temporal sampling) light curves. The larger uncertainty in $\mathcal{R}_{U V}$ for the Keck/SDSS SNe is due to the combination of slightly lower-S/N spectra and larger values of $\mathcal{R}_{U V}$ (for spectra with a fixed $\mathrm{S} / \mathrm{N}$, one naturally expects a larger uncertainty for larger values of $\left.\mathcal{R}_{U V}\right)$.

Examining Figure 12, we immediately notice that for

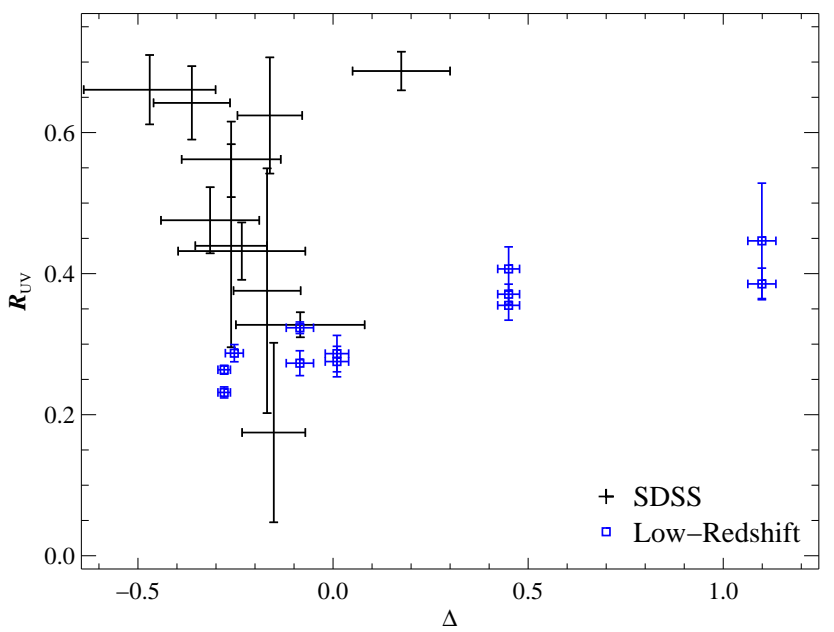

FIG. 12.- Relationship between the UV ratio $\left(\mathcal{R}_{U V}\right)$ and $\Delta$. The black points represent the SNe in our Keck/SDSS sample. The blue squares are the low-redshift sample from FFJ. The Keck/SDSS SNe are obviously offset to higher values of $\mathcal{R}_{U V}$, the result of the UV excess.

a given $\Delta$, the Keck/SDSS sample has a higher value of $\mathcal{R}_{U V}$. This is expected in view of the UV excess in the SDSS-II sample noted above; the UV excess is more prominent at shorter wavelengths. Unfortunately, the small range of $\Delta$ for the Keck/SDSS sample does not allow us to determine whether the trend seen at low redshift (i.e., $\mathcal{R}_{U V}$ increases with $\Delta$ ) also exists at high redshift.

As a test of using $\mathcal{R}_{U V}$ as a luminosity indicator, we wish to determine $M_{V}$ for each Keck/SDSS SN Ia and compare that to $\mathcal{R}_{U V}$. To find $M_{V}$, we first measure the peak apparent $r$ or $i$ magnitude (depending on the redshift of the SN) using MLCS template light curves fit to the photometry in that single band. Then, using the concordance cosmology $\left(H_{0}=70 \mathrm{~km} \mathrm{~s}^{-1} \mathrm{Mpc}^{-1}\right.$, $\Omega_{m}=0.3, \Omega_{\Lambda}=0.7$, and $w=-1$ ), we can determine the distance modulus $(\mu)$ of each SN. From our MLCS fits and Schlegel et al. (1998), we are able to determine the host-galaxy and Milky Way reddenings, $A_{V \text { host }}$ and $A_{X, \mathrm{MW}}$, respectively. Finally, from our MLCS fits, we are also able to derive a $K$-correction, $\mathrm{K}_{X V}$, where $X V$ denotes a correction from observed-band $X$ to rest-frame $V$. Then, using the equation

$$
M_{V}=X_{\max }-\mu-A_{V, \text { host }}-A_{V, \mathrm{MW}}-\mathrm{K}_{X V},
$$

where $X$ denotes either the $r$ or $i$ band, we are able to determine the absolute visual magnitude for each SN. Note that there are assumptions made regarding the $K$ corrections which may not be appropriate for our sample given the differences between the spectra of low-redshift and Keck/SDSS SNe seen above. However, the restframe $V$ band is relatively free of these differences. Another assumption is that the MLCS-measured value for $A_{V}$ applies to our sample given the observed color differences between the high and low-redshift samples. However, uncertainties in $A_{V}$ and $\mathrm{K}_{r V}$ are typically $\sim 0.05$ and $0.02 \mathrm{mag}$, respectively, which are generally smaller than the errors associated with the determination of $r_{\max }$ and $\mu$. 


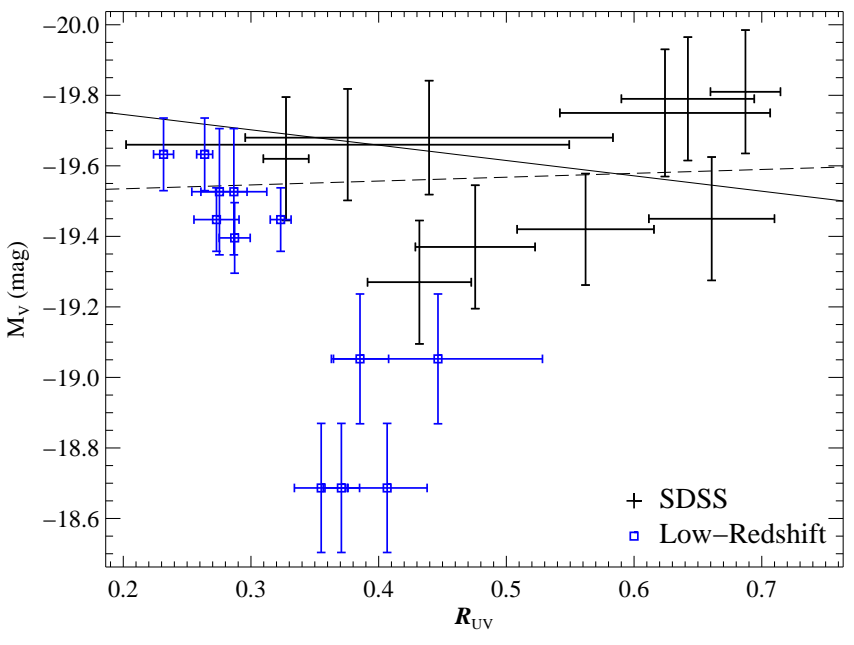

FIG. 13. - Relationship between the UV ratio $\left(\mathcal{R}_{U V}\right)$ and $M_{V}$ at peak brightness. The black points represent the $\mathrm{SNe}$ in our Keck/SDSS sample. The blue squares are the low-redshift sample from FFJ. There are multiple measurements for some low-redshift $\mathrm{SNe}$ (from different spectra over $-3<t<3$ days). Each lowredshift SN has a unique value of $M_{V}$. The solid black line is the relationship given in Equation 13 The dashed black line is the relationship given in Equation 14 including the low-redshift data with $M_{V}<-19.0 \mathrm{mag}$, corresponding to smaller $\Delta$. The Keck/SDSS SNe follow a different trend than the low-redshift $\mathrm{SNe}$ that cannot be attributed to only the UV excess, which would shift the SNe to higher values of $\mathcal{R}_{U V}$ (and potentially change the slope slightly). However, it is possible that all SNe with $M_{V}<$ -19.0 mag follow a simple relationship.

We compare the values of $\mathcal{R}_{U V}$ and $M_{V}$ for the Keck/SDSS and low-redshift SNe in Figure 13 As found by FFJ, the low-redshift $\mathrm{SNe}$ follow the relationship

$$
M_{V}(t=0)=-19.366+5.686\left(\mathcal{R}_{U V}-0.3\right) \mathrm{mag},
$$

where we have adjusted $M_{V}$ for the SNe to match $H_{0}=$ $70 \mathrm{~km} \mathrm{~s}^{-1} \mathrm{Mpc}^{-1}$. It is obvious that the trend seen in the low-redshift sample is not present in the Keck/SDSS sample. One might expect a shift of the trend to higher $\mathcal{R}_{U V}$, but a similar slope, if the UV excess is equal for all Keck/SDSS SNe. However, fitting all Keck/SDSS SNe with spectra that reach rest-frame $2750 \AA$, we find

$$
M_{V}(t=0)=-19.702+0.435\left(\mathcal{R}_{U V}-0.3\right) \mathrm{mag},
$$

which is much shallower than the low-redshift trend. The fit corresponding to Equation 13 has $\chi^{2} /$ dof $=15.9 / 11$.

The low-redshift sample contains only six $\mathrm{SNe}$, while the Keck/SDSS sample consists of SNe from a small range of $M_{V}$ (and $\Delta$ ). Four of the low-redshift SNe appear to be consistent with the relationship derived from the Keck/SDSS SNe, while two (those with the highest value of $\Delta$, and corresponding to the five low-redshift points with $M_{V}>-19.1 \mathrm{mag}$ in Figure 13) are inconsistent. If we examine all $\mathrm{SNe}$ (at both low and high redshift) with $M_{V}<-19.1 \mathrm{mag}$, we determine

$$
M_{V}(t=0)=-19.546-0.110\left(\mathcal{R}_{U V}-0.3\right) \mathrm{mag},
$$

with $\chi^{2} /$ dof $=25.9 / 18$. The values for the linear fits for Equations 12 14 and corresponding uncertainties are presented in Table 3 .

Both samples must be expanded to determine whether (a) these trends are real within their own populations,
TABLE 3

$M_{V}-\mathcal{R}_{U V}$ Linear Fits

\begin{tabular}{ccr}
\hline \hline \multicolumn{3}{c}{$M_{V}(t=0)=A+B\left(\mathcal{R}_{U V}-0.3\right)$} \\
Equation
\end{tabular}

(b) the low and high-redshift samples have truly different relationships, and (c) subsamples of SNe form groups in the $\mathcal{R}_{U V}-M_{V}$ plane.

\section{DISCUSSION}

To summarize our results, we find (Section 3) that the intermediate-redshift Keck/SDSS sample has significantly more flux $(>20 \%)$ in the near-UV $(\lambda<3400 \AA)$ relative to a low-redshift sample. This mismatch has significant implications for measuring distances with SNe Ia (Section 44). In Section 5, we examine subsamples of the Keck/SDSS sample to determine if there were any obvious correlations between spectral features and redshift, host properties, and light-curve shape, respectively. We find that the UV SED of the Keck/SDSS sample does not depend on redshift, and there are no significant differences that correlate with host-galaxy mass or light-curve shape.

We now investigate possible systematic errors which could create a UV mismatch. We also discuss this result in the context of $\mathrm{SN}$ evolution.

\subsection{Possible Systematic Errors}

There are many possible systematic errors associated with the spectral shape of the SNe Ia in our study. They can be separated into three categories: sample selection, spectral reductions, and post-processing techniques. We address each of these in turn.

\subsubsection{Sample Selection}

The above results rely on the assumption that the Keck/SDSS and low-redshift samples were randomly selected from their respective populations. Here we discuss a possible source of systematic uncertainty from selection bias. If the Keck/SDSS sample presented here is not representative of high-redshift $\mathrm{SNe}$ Ia, then any conclusions drawn from this sample are possibly incorrect. Similarly, if the sample used to create the low-redshift composite spectrum is biased relative to the sample of $\mathrm{SNe}$ used in a cosmological analysis, the same caveats apply.

If the Keck/SDSS sample is not a random subset of the larger spectroscopic SDSS-II sample, then we would expect there to be some additional observational evidence besides a UV excess. In Figure 14, we compare the light-curve shape parameters of our subsample to the 99 SNe Ia used in the first SDSS-II cosmological analysis (K09). Performing a Kolmogoroff-Smirnov (K-S) test, we find that the two samples are not significantly different, with a $p$-value of 0.348 . Therefore, the Keck/SDSS sample appears to be representative (in terms of light-curve shape) of the overall SDSS-II spectroscopic sample.

In Figure 15] we show the measured value of $\Delta$ for each $\mathrm{SN}$ as a function of redshift. There is no real indication of Malmquist bias. A best-fit line to the $\Delta$ distribution 


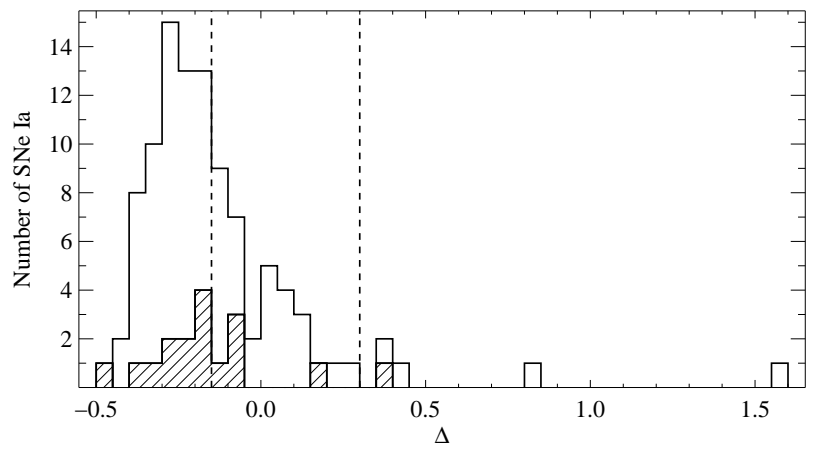

FIG. 14.- Histogram of the $\Delta$ distribution of the SDSS-II SNe Ia from the first SDSS-II cosmological analysis and the subsample presented in this paper (dashed histogram). The dashed lines mark the regions of overluminous $(\Delta<-0.15)$, normal $(-0.15<\Delta<$ $0.3)$, and underluminous $(\Delta>0.3) \mathrm{SNe}$, as defined by Jha et al. (2007) and shown in Figure 2

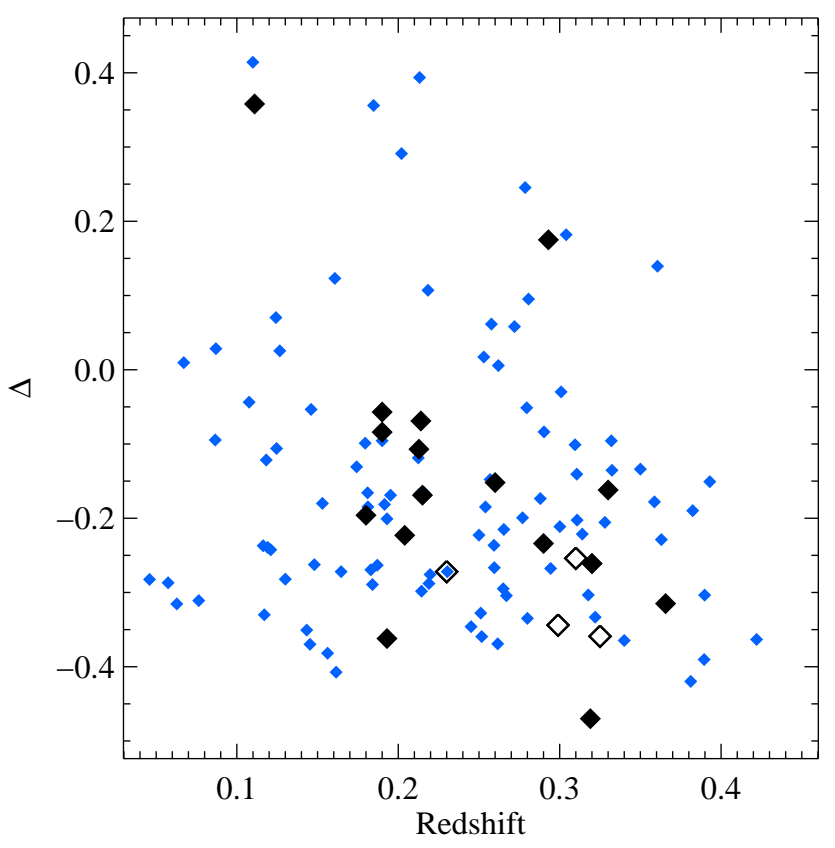

FIG. 15.- The $\Delta$ distribution of Keck/SDSS SNe Ia presented in this paper as a function of redshift. The filled black diamonds are the Keck/SDSS SNe in the Nominal sample. The open black diamonds are the Keck/SDSS SNe rejected from the Nominal sample. The smaller blue diamonds are the full SDSS-II first-year sample. A K-S test shows that the Keck/SDSS and full SDSS-II samples are consistent.

of the Nominal sample with redshift is $1.1 \sigma$ from zero slope. This, combined with the changing demographics of the SN population and the effect on average lightcurve shape (Howell et al. 2007), suggests that there is no significant Malmquist bias.

The full, photometric SDSS-II sample is certainly Malmquist biased, but the SDSS-II sample should be nearly unbiased to $z \approx 0.3$ for discoveries (as opposed to spectroscopic completeness, which is limited to $z \approx$ 0.15 ; K09); the Keck/SDSS sample may be similarly Malmquist biased, although we do not strongly detect
TABLE 4 SDSS-II $g$-Band Data/Model Properties

\begin{tabular}{lcccc}
\hline \hline Redshift Range & $0-0.1$ & $0.1-0.2$ & $0.2-0.3$ & $>0.3$ \\
Number of SNe & 10 & 34 & 35 & 23 \\
$\langle z\rangle$ & 0.072 & 0.153 & 0.250 & 0.349 \\
$\left\langle p_{\mathrm{UV}}\right\rangle$ & 0.20 & 0.15 & -0.31 & -0.38 \\
$\langle\lambda\rangle^{\mathrm{a}}$ & 4760 & 4420 & 4080 & 3780 \\
\hline
\end{tabular}

a Approximate rest-frame wavelength of the center of the $g$ band at the average redshift for the redshift bin.

it.

Besides a simple Malmquist bias where we are selecting more-luminous $\mathrm{SNe}$, we may be selecting bluer $\mathrm{SNe}$ than the typical high-redshift SN Ia included in cosmological analyses. This is particularly problematic for $z>1 \mathrm{SNe}$ Ia where searches are usually performed in the rest-frame near-UV (but photometrically followed in redder bands). For the SDSS-II sample, the search is performed in gri, avoiding the near-UV, which should reduce such a bias. The difference between $g-r$ color of the Keck/SDSS and low-redshift composite spectra is $\Delta(g-r)=0.1 \mathrm{mag}$ at $z=0.35$, and less for lower redshifts. Therefore, it is highly unlikely that such a bias is directly affecting our sample.

Nevertheless, the Keck/SDSS sample has an excess of rest-frame UV flux relative to the low-redshift sample, which in turn has an excess of rest-frame UV flux relative to the full SDSS-II sample (K09). Examining the $g$-band MLCS2k2 light-curve fits for the K09 sample, we can assign a value, which we call $p_{\mathrm{UV}}$, of $-1,0$, or 1 to light curves that are below, consistent with, and above the MLCS2k2 $g$-band model, respectively. Our results are shown in Table 4. With increasing redshift, corresponding to bluer wavelengths, the $g$-band data of the SDSS-II sample go from being above the model to significantly below the model. The 12 SNe from the Keck/SDSS sample that are also in the K09 sample, on the other hand, have $\left\langle p_{\mathrm{UV}}\right\rangle=0.23$ (indicating that the sample tends to be above the model), with $\langle z\rangle=0.250$. For the $0.2-$ 0.3 redshift bin, the K09 has $\langle z\rangle=0.250$ (the same as the Keck/SDSS sample within the K09 sample), but $\left\langle p_{\mathrm{UV}}\right\rangle=-0.31$. Although there is no obvious color selection bias that causes this effect, the differences appear to be real. It is therefore important to examine other differences between the various samples.

The low-redshift sample has few UV spectra, and it is possible that these $\mathrm{SNe}$ are not representative of lowredshift SNe Ia. The UV portion of the maximum-light composite spectrum consists of 15 spectra from $7 \mathrm{SNe}$ (FFJ; SNe 1980N, 1981B, 1986G, 1990N, 1991T, 1992A, and $2005 \mathrm{cf}$ ), all observed with IUE or Swift. The lowredshift composite spectrum begins to deviate from the Keck/SDSS composite spectrum at $\sim 4000 \AA$, which is easily observed from the ground (and not included in any $I U E$ spectra), is not heavily affected by the atmosphere, and is sampled by many spectra. We therefore do not think that the small sample of low-redshift UV spectra is biasing our results. Although a larger number of lowredshift UV spectra of SNe Ia is desperately needed (and is being addressed by our Swift progran[23] and comple-

23 PI Filippenko, Programs 04047, 5080130; PI Foley, Program 6090689 
mentary HST program [24, a biased low-redshift set of SNe Ia having available UV spectra does not appear to be the cause of the UV difference.

Alternatively, the low-redshift and Keck/SDSS samples might have intrinsic differences that may cause discrepancies in UV spectra but not show differences in light-curve shape. To properly compare the samples, we must consider the intrinsic differences of the surveys that discover $\mathrm{SNe}$ at low redshift compared to that of SDSS-II. The predominant method of finding SNe at low redshift is through targeted surveys, where a sample of galaxies is searched continually. In particular, this is the method used by the Lick Observatory Supernova Search (LOSS; Filippenko et al. 2001; Filippenko 2005a), from which a large fraction of the low-redshift SNe Ia come. SDSS-II, on the other hand, is an untargeted survey, observing the same part of the sky continually, but not targeting particular galaxies. This means that the sample demographics are different. Although most of the stars in an untargeted survey are in galaxies similar to those searched by targeted surveys, so they are not dramatically different, there may still be a significant difference in the host-galaxy population. Specifically, the host galaxies of the Keck/SDSS sample have a median mass of $(2.6-3.6) \times 10^{9} \mathrm{M}_{\odot}$ (depending on how one treats the host-galaxy nondetections), while the average masses for $\mathrm{E}, \mathrm{Sb}$, and Irr galaxies from LOSS, from which a significant number of the low-redshift sample come, are 16.0, 6.4 , and $2.4 \times 10^{10} \mathrm{M}_{\odot}$ (Leaman et al. 2010). Therefore, the average SN Ia from the Keck/SDSS sample is hosted in a galaxy that is probably $\sim 0.1$ times the mass of the average irregular galaxy and $\sim 0.02$ times the mass of the average elliptical galaxy in the low-redshift sample. Given the mild differences found in the spectra of SNe hosted in low and high-mass hosts (see Section 5.2) and the correlation between host-galaxy mass and Hubble residuals (Kelly et al. 2010; Lampeitl et al. 2010b; Sullivan et al. 2010), we consider variations imprinted on $\mathrm{SNe}$ Ia by their environments as a possible cause of the difference. However, the Keck/SDSS sample is a subset of the SDSS-II sample, and it is unclear how it could have significantly different average host properties. Future untargeted, low-redshift SN samples from surveys such as the Palomar Transient Factory (PTF), La Silla-QUEST, SkyMapper, or Pan-STARRS should provide SNe with similar host-galaxy properties as the high-redshift SNe. (However, the low-redshift sample of Cooke et al. 2011 primarily came from PTF and showed similar UV flux as our low-redshift sample.)

A second difference between the searches is that those at low redshift tend to be done in a single band (often unfiltered, which for LOSS is similar to the $R$ band), while the SDSS-II search is performed in gri. The SDSSII search requires a detection in two of the filters. At $z \approx 0.25$, these are typically $r$ and $i$. It is therefore unlikely that the Keck/SDSS sample is biased to select significantly bluer SNe at discovery than for the low-redshift sample. In a typical exposure, the SDSS-II SN survey achieved a $50 \%$ detection limit of $g=22.7 \mathrm{mag}$, which is $\sim 0.5$ mag deeper than the typical $z=0.35 \mathrm{SN}$ Ia at peak. As mentioned above, the difference between the $g-r$ color for the Keck/SDSS and low-redshift compos-

${ }^{24}$ PI Ellis, Programs 11721 and 12298; PI Foley, Program 12592. ite magnitudes is $\Delta(g-r)=0.10 \mathrm{mag}$ at $z=0.35$ (and $\Delta(g-r)<0.2 \mathrm{mag}$ until $z \approx 0.55$ ), so we should not be significantly affected by this potential bias. Additionally, the rest-frame $g-r$ colors of the Nominal and low-redshift composite spectra is only 0.069 mag.

Examining Figure 2, we see that the Keck/SDSS sample is predominantly composed of SNe with broad light curves (i.e., low $\Delta$ ). Although the full low-redshift and Keck/SDSS samples have different demographics, it is important to point out that (a) this is a natural consequence of the evolving demographics of SNe Ia with redshift (e.g., Howell et al. 2007), and (b) the low-redshift sample we have used to generate the comparison lowredshift composite spectrum has very similar properties to that of the Keck/SDSS sample (as demonstrated by the comparison of phase and $\Delta$ in Figure 51). Consequently, differences in the spectra as a result of different photometric and phase properties should be largely mitigated and not biasing our findings. However, note that we draw some conclusions in later sections based on the full low-redshift photometric sample which may not show a similar trend.

\subsubsection{Spectral Reductions}

Another set of possible systematic errors is associated with the observations and reduction process. The two major sources of systematic error would be the systematic "reddening" of the spectra as the result of the spectroscopic slit not being aligned along the parallactic angle (Filippenko 1982) and incorrect flux calibration.

All of the Keck observations were taken within $5^{\circ}$ of the parallactic angle to preserve the intrinsic SED of the SN spectrum. Furthermore, the observations taken in 2007 were with the ADC in place, reducing the effective atmospheric dispersion to a negligible amount for all but the highest airmasses. Moreover, if significant atmospheric dispersion were present at high airmass, the blue flux should decrease more than the red, given that the $\mathrm{SNe}$ were centered in the slit while using the red-sensitive LRIS guide camera - yet this is the opposite of the observed difference between the low and high-redshift samples. (Note that the standard stars were observed in the same manner as the SNe.) In addition, the low-redshift UV spectra were obtained from space, where atmospheric dispersion does not affect the observations.

The second possibility is that the flux calibration of the SN spectra was incorrect, causing an overestimate of the bluest fluxes. However, the spectra were obtained over three seasons on several nights. Therefore, any fluxcalibration error must have occurred for these temporally separated observations. The Keck/SDSS SN spectra were also reduced in exactly the same manner as that of the optical spectra from the low-redshift sample (and similar in methodology for the UV spectra), with all Keck/SDSS SN spectra and a significant number of low-redshift SN spectra reduced by the same person (R.J.F.). If there is an inherent flux-calibration error for all spectra, this would have been observed in the lowredshift sample as well. A second set of spectral reductions was performed by another person (Ryan Chornock) with similar results. Additionally, the spectra obtained in 2005 were observed with polarimetry optics, producing two spectra for each object that were reduced with completely separate calibrations, yet they always agreed 
within the uncertainties. A final argument against a fluxcalibration error is that these errors would be present in the observer frame, not in the rest frame where the difference is apparent. Since our spectra span a range in redshift, the errors should be present at varying wavelengths and not correspond to the same rest-frame wavelengths. However, when separating our sample by redshift, we see no difference (see Section 5.1). Because of our meticulous flux calibration with multiple decades of testing, flux-calibration errors seem unlikely.

\subsubsection{Post-Processing Techniques}

A final candidate for systematic errors resides in the post-processing techniques performed on the spectra. The possibilities include incorrect extinction corrections, galaxy-contamination corrections, and creation of the composite spectra.

There are two separate extinction corrections applied to the spectra. The first is a Milky Way extinction correction, using the dust maps of Schlegel et al. (1998). The extinction measurements from these maps are typically quite good. Furthermore, the Keck/SDSS SNe tend to come from low-extinction regions $(E(B-V) \lesssim$ $0.05 \mathrm{mag})$. It is therefore unlikely that the Milky Way extinction corrections are sufficiently erroneous to significantly influence the measured flux.

The second extinction correction is to account for the line-of-sight extinction due to host-galaxy dust. The extinction has been estimated during the MLCS fitting. For our Keck/SDSS sample, the estimates are typically low (median value for the Nominal sample is $A_{V}=0.04 \mathrm{mag}$ and the maximum value is $A_{V}=0.43 \mathrm{mag}$ ). However, the reddening is determined by comparing the highredshift SN light curves to template light curves derived from a low-redshift training set. Consequently, if there is a difference between the low and high-redshift samples, this estimate may be incorrect. On the other hand, since the extinction estimates are low, we are making small corrections to the spectra, and it is difficult to explain how these small corrections could create the large UV excess in the Keck/SDSS composite spectrum. Finally, Foley et al. (2008a) showed that composite spectra formed from only low-extinction, uncorrected spectra and composite spectra formed from both low and high-extinction, extinction-corrected spectra are consistent with each other.

The light curves of the Keck/SDSS sample were fit with $R_{V}=1.9$, the best-fit value found for the full SDSS-II sample25. However, this value of $R_{V}$ is low compared to that seen in the Milky Way (Schlegel et al. 1998), which may be partially the result of mixing SNe Ia with different intrinsic colors (Foley \& Kasen 2011). We have performed a separate set of light-curve fits with $R_{V}=3.1$. Since the value of $A_{V \text {,host }}$ is low for the Keck/SDSS SNe, changing the value of $R_{V}$ did not dramatically alter the values of $A_{V \text {,host }}$. In Figure 16, we show the Keck/SDSS composite spectrum presented in Figure 5 (created from the Nominal sample with $R_{V}=1.9$ for all light-curve fits and our extinction corrections) and a composite spectrum created from the Nominal sample with $R_{V}=3.1$ for all light-curve fits and extinction corrections. These

25 This value of $R_{V}$ was determined by comparing the SDSS-II photometry to simulations; see Section 7.2 of Kessler et al. (2009a).

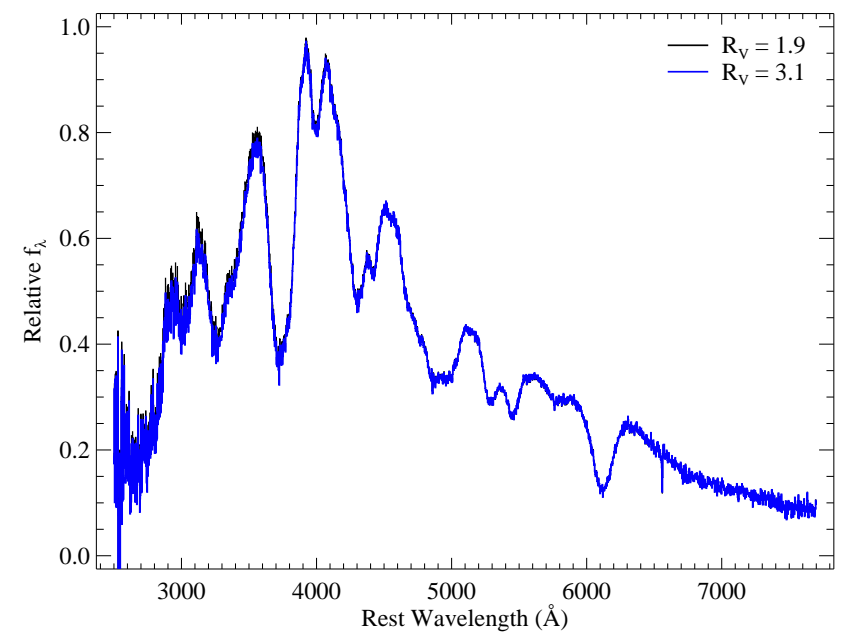

FIG. 16. - Composite spectra from Keck/SDSS SNe in the Nominal sample with $R_{V}=1.9$ (black curve) and $R_{V}=3.1$ (blue curve) for all light-curve fits and extinction corrections. The spectra are nearly indistinguishable, indicating that the choice of $R_{V}$ does not affect the UV excess.

two spectra are created from the same sample of SNe, and the only difference is in the value of $R_{V}$ and derived $A_{V \text {,host }}$. Although the different value for $R_{V}$ does change the SED of the composite spectrum slightly, it is not a sufficiently large difference to compensate for the UV excess.

As shown in Figure 19, the galaxy-contamination correction appears to be quite good for the observer-frame gri bands. These measurements do not include the observer-frame near-UV region, where the rest-frame UV is observed. However, the galaxy spectra are determined from ugriz photometry, which does include the observer-frame near-UV region, and other than at $0.27<z<0.33$, the reconstruction of galaxy SEDs is very good (Blanton et al. 2003). Finally, as seen in Figure 4, the composite spectra created from the full Keck/SDSS sample presented in this paper and from the sample of SNe with little or no galaxy contamination are consistent. It is thus unlikely that incorrect galaxy contamination causes the UV excess.

The final possible source of post-processing systematic error is related to creating the composite spectra. Since the low-redshift and Keck/SDSS composite spectra were created in exactly the same way, systematic differences should be minimal. The one major possible systematic error associated with creating the composite spectra comes from splicing some low-redshift spectra together. Unlike with the Keck/SDSS spectra, which all contain a large overlapping rest-frame wavelength range, some lowredshift spectra are completely disjoint. Accordingly, we splice the spectra together using an iterative process, creating composite spectra, rescaling spectra, and recreating composite spectra until all spectra are added. This is especially important for those particular UV spectra whose longest wavelengths are $\sim 3200 \AA$.

For most composite spectra, the overlap between the UV and ground-based optical spectra is $\sim 100 \AA$. Consequently, there may be an offset between the UV and optical portions of the composite spectrum at $\sim 3200 \AA$ (the 


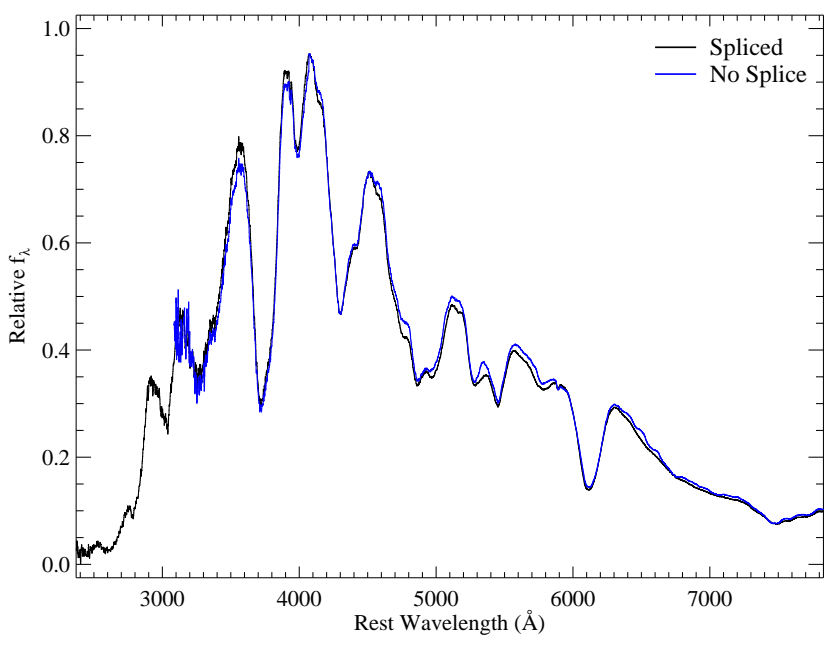

Fig. 17.- Low-redshift composite spectra with (black curve) and without (blue curve) splicing spectra (as described in the text). The small differences between the spectra are mainly the result of slightly different samples and the iterative scaling of each spectrum allowed in the splicing method. The spectra are extremely similar for the overlapping wavelengths, indicating that splicing the UV spectra to the optical spectra does not affect the flux at the shortest optical wavelengths.

number of spectra for the composite spectra as a function of wavelength can be seen in Figure 50). We have created a composite spectrum without splicing any spectra together (using only the SNe that overlap over the same $1000 \AA$ region), and compare in Figure [17 that spectrum to our composite spectrum consisting of all spectra. From this figure we see that the method of splicing spectra together does not significantly change the composite spectrum over the wavelength region covered by both spectra. Figure 17 also shows that the UV spectra appear to be spliced properly, creating the correct SED. However, we cannot rule out the possibility of errors at the ends of our spectra combining to incorrectly set the scaling of the UV spectra. Regardless of these difficulties, there are differences between the Keck/SDSS and low-redshift composite spectra at wavelengths longer than $3200 \AA$, indicating that there are probably some real differences between these samples. With additional UVoptical spectra obtained at exactly the same epoch, we may be able to address this further.

\subsection{Supernova Evolution}

No apparent systematic error is creating the UV excess for the Keck/SDSS SN Ia sample. We now investigate the possible physical reasons for a UV excess in the high-redshift SNe Ia compared to low-redshift SNe Ia. Changes in SNe Ia with redshift are typically called "evolution," but it is possible that the sample demographics are simply shifting with redshift (see Foley et al. 2008a and Leibundgut 2001 for discussions). We will not distinguish between these scenarios here, but we point out that if the cause of the difference is related to host-galaxy mass (or star-formation rate, etc.), which is compounded by the differences between targeted (at low redshift) and untargeted (at high redshift) SN searches, this would not be considered evolution.
K09 found that including and excluding the observed $u$ band for nine low-redshift $(0.04<z<0.09)$ SDSSII SNe resulted in the same distance modulus offset as found for the rest of the SDSS-II sample when including and excluding the rest-frame $U$ band. The non-SDSSII low-redshift sample $(z \approx 0.03$ for most $\mathrm{SNe})$ did not show this offset; if evolution is causing a difference in the SEDs, the evolution must occur quickly and recently.

There are several models for SNe Ia evolving with redshift as the result of changing progenitor metallicity (e.g., Höflich et al. 1998; Lentz et al. 2000; Sauer et al. 2008). A major conclusion of these studies is that the optical spectra will have relatively small differences, while the UV will show a significant difference. Unfortunately, there is no consensus as to whether the UV flux will increase or decrease with increasing metallicity.

The models of Höflich et al. (1998) indicate that UV excess is expected with higher progenitor metallicity. Lentz et al. (2000), however, showed model spectra where UV excess is the result of lower progenitor metallicity. The differences are the result of differing density structures (Lentz et al. 2000; Domínguez et al. 2001). It is beyond the scope of this study to determine which model is more accurate. Although other factors such as the progenitor $\mathrm{C} / \mathrm{O}$ ratio may influence the $\mathrm{UV}$ flux, the difference in UV spectra appears to be broadly consistent with models of differing metallicity.

The slight apparent difference between SN Ia UV spectra from SNe with active/low-mass and passive/highmass host galaxies indicates that $\mathrm{SNe}$ Ia with different progenitors or delay times may result in different explosions despite having similar light-curve shapes. It is necessary to obtain further observations to increase the sample size and see if this trend persists.

There is a clear difference in the relationship between $\mathcal{R}_{U V}$ and $M_{V}$ for the low-redshift and Keck/SDSS samples. It is possible that this is a sign of evolution. However, neither the low nor high redshifts have been adequately sampled in the full $M_{V}-\Delta$ plane. We must therefore expand these samples to determine if these correlations persist. However, at this time the relationship between $\mathcal{R}_{U V}$ and $M_{V}$ found for the low-redshift sample by FFJ clearly should not be used for determining the peak luminosity of a high-redshift SN Ia.

When comparing the Keck/SDSS and low-redshift samples, we have controlled for most observables. In particular, the composite spectra shown in Figure [5 have the same average phase and light-curve shape. The one obvious observable which is vastly different between the samples is host-galaxy mass. As we discussed above, the average host for an SN in the low-redshift sample is probably 10-50 times more massive than that of an SN in the Keck/SDSS sample. Given the similarity in other parameters, we wonder if such a difference is the dominant factor in the observed differences. Future samples at both low and high redshifts should be able to answer this question.

\section{CONCLUSIONS}

Using samples of high-quality low-redshift and intermediate-redshift SN Ia spectra, we have provided evidence of differences between the SNe Ia in these samples. By using SN and host-galaxy photometry, we are able to properly separate the SN and host-galaxy spectra. 
Constructing a composite spectrum from 17 of our hostgalaxy contamination-corrected Keck/SDSS SNe, we see that the intermediate-redshift $\mathrm{SNe}$ have $>20 \%$ more UV flux $(\lambda<3400 \AA)$ of their low-redshift counterparts. This UV excess is also apparent in synthesized broadband colors at high significance. After an exhaustive search for systematic errors, we determined that poor spectral reductions, flawed post-processing techniques, or Malmquist-biased sample selection are unlikely to be creating this effect. It is possible, however, that a combination of several small systematic effects creates the UV excess. Despite having otherwise very similar properties, the UV flux of the Keck/SDSS sample does not appear to be representative of the larger SDSS-II sample.

We have also shown that there may be a slight difference in the UV spectra of SNe from low-mass and highmass host galaxies (or, alternatively, those that are actively forming stars versus those that are not). This difference is currently of low statistical significance and requires larger samples to further improve confidence in the result. In particular, SNe with more massive hosts must be probed at high redshift. If SNe Ia originating from different stellar populations have similar optical light-curve shapes but different UV spectra, observing the UV spectra of SNe Ia may offer a new window to understanding the progenitors of SNe Ia and could potentially improve distance estimates. Several studies have now found a significant correlation between host-galaxy mass, morphology, and star-formation rate with Hubble residuals. Detailed analysis of spectra may reveal the physical cause of these correlations.

The UV ratio, $\mathcal{R}_{U V}$, has a substantially different correlation with $M_{V}$ in the low-redshift and Keck/SDSS samples. Since these samples are relatively small and that of Keck/SDSS has little diversity in SNe Ia, it is still unclear whether this difference is inherent to low and high-redshift samples or is the result of insufficient samples. More observations at both low and high redshifts are necessary to make this determination.

The differences in the UV spectra of low and highredshift SNe Ia may be the result of SN Ia evolution. Future observations and careful modeling are necessary to determine the magnitude and implications of this possibility. However, a more reasonable explanation is that the low-redshift and high-redshift samples differ in some significant way. We have accounted for phase and lightcurve shape when comparing these samples, but given the heterogeneous nature of (and incomplete information for) the low-redshift sample, we were unable to account for additional potential differences such as host-galaxy properties. Since the low-redshift SNe Ia were discovered through surveys which target the most luminous galaxies in the nearby Universe and the SDSS-II survey was untargeted, it is likely that the host-galaxy properties for these samples are quite different.

The UV excess seen in the Keck/SDSS sample is not seen in the full SDSS-II sample, as inferred from their light curve (K09). In fact, the full SDSS-II sample is, on average, UV deficient relative to the low-redshift sample. There is no clear reason for this difference, and it may simply be the result of a relatively small sample (although the Keck/SDSS sample represents $12 \%$ of the full K09 SDSS-II sample).

A mismatch in the UV SEDs of SNe Ia like that seen between the Keck/SDSS and the low-redshift samples has important implications for the estimated distances to all SNe Ia. Although the Keck/SDSS sample is relatively large, it still is not fully representative of the larger SDSS-II sample. This indicates that there is a significant scatter in the UV properties of SNe Ia (at least at intermediate redshift). A simplistic treatment of this flux difference suggest that the incorrect normalization of the peak luminosity (an offset in $M_{V \text {,peak for a }}$ given light-curve shape) and an incorrect determination of the host-galaxy extinction (changing the measurement of $A_{V}$ ) should both contribute to an underestimate of the correct distance for an SN Ia. Fitting multi-band data that cover the rest-frame UV and optical will tend to cause bluer SNe Ia to have a higher measured peak luminosity than reality, causing an opposite (and dominant) effect. This effect is constrained to the (near) UV of an SN Ia SED, and by ignoring this region, one should obtain an unbiased (by this effect) distance estimate.

A light-curve fitter trained on a low-redshift sample (MLCS2k2) and applied to high-redshift samples would produce significantly different distance measurements if the rest-frame $U$ band were included or excluded. This was not seen with a light-curve fitter which was trained on the full (low and high-redshift) sample (SALT2). If the cause of the $U$-band anomaly was a mismatch in the UV SEDs for the low and high-redshift samples, SALT2 would have a model SED close to both the low and highredshift samples and differences would be minimized.

For the James Webb Space Telescope (JWST), Euclid, and the future potential Wide-Field InfraRed Survey Telescope (WFIRST) mission, which will observe SNe in the near-infrared, there should be a relatively small systematic color bias to a redshift of $\sim 2.5$. Since current designs do not plan to observe SNe Ia beyond that redshift, differences seen in our sample should have a relatively small effect on JWST, Euclid, or WFIRST measurements.

R.J.F. is supported by a Clay Fellowship.

The SDSS-II SN team supplied targets, photometry, and light-curve fits for this project; in particular, G. Miknaitis provided some of the necessary data and discussions. We thank J. M. Silverman for help with some of the observations, and R. Chornock for useful discussions and for performing additional spectral reductions of several objects. We are grateful to M. Blanton for discussions about the kcorrect software and J. Bloom for insightful comments on an early draft of this work. We thank the referee, M. Stritzinger, for helpful comments. The spectra of intermediate-redshift SNe in this study were obtained at the W. M. Keck Observatory, which is operated as a scientific partnership among the California Institute of Technology, the University of California, and the National Aeronautics and Space Administration (NASA); the Observatory was made possible by the generous financial support of the W. M. Keck Foundation. The spectra of low-redshift SNe in this study were obtained with the $3 \mathrm{~m}$ Shane telescope at Lick Observatory, which is owned and operated by the University of California. We thank the Keck and Lick staffs for their assistance with the observations.

A.V.F. is grateful for the hospitality of the Aspen 
Center for Physics, where this paper was finalized during the January 2012 program on "The Physics of Astronomical Transients." This research was made possible by NSF grants AST-0443378, AST-0507475, AST0607485, and AST-0908886, as well as by NSF CAREER award AST-0847157 to S.W.J. at Rutgers University. Additional support was provided by U.S. Department of Energy (DOE) grant DE-FG02-08ER41562 and by the TABASGO Foundation. We also acknowledge funding from NASA/HST grant GO-10182 from the Space Telescope Science Institute, which is operated by the Association of Universities for Research in Astronomy, Inc., under NASA contract NAS5-26555.

Funding for the SDSS and SDSS-II has been provided by the Alfred P. Sloan Foundation, the Participating Institutions, NSF, DOE, NASA, the Japanese Monbukagakusho, the Max Planck Society, and the Higher Education Funding Council for England. The SDSS Web Site is http://www.sdss.org/

The SDSS is managed by the Astrophysical Research
Consortium for the Participating Institutions. The Participating Institutions are the American Museum of Natural History, Astrophysical Institute Potsdam, University of Basel, University of Cambridge, Case Western Reserve University, University of Chicago, Drexel University, Fermilab, the Institute for Advanced Study, the Japan Participation Group, Johns Hopkins University, the Joint Institute for Nuclear Astrophysics, the Kavli Institute for Particle Astrophysics and Cosmology, the Korean Scientist Group, the Chinese Academy of Sciences (LAMOST), Los Alamos National Laboratory, the Max-Planck-Institute for Astronomy (MPIA), the MaxPlanck-Institute for Astrophysics (MPA), New Mexico State University, Ohio State University, University of Pittsburgh, University of Portsmouth, Princeton University, the United States Naval Observatory, and the University of Washington.

Facilities: Keck I (LRIS), Magellan:Clay (MagE), Sloan Digital Sky Survey

\section{REFERENCES}

Adelman-McCarthy, J. K., et al. 2008, ApJS, 175, 297

Barentine, J., et al. 2005, Central Bureau Electronic Telegrams, 280,1

Bassett, B., et al. 2007a, Central Bureau Electronic Telegrams, 1102,1

—. 2007b, Central Bureau Electronic Telegrams, 1104, 1

—. 2007c, Central Bureau Electronic Telegrams, 1139, 1

- 2006, Central Bureau Electronic Telegrams, 762, 1

Blanton, M. R., et al. 2003, AJ, 125, 2348

Blanton, M. R., \& Roweis, S. 2007, AJ, 133, 734

Blondin, S., \& Tonry, J. L. 2007, ApJ, 666, 1024

Bufano, F., et al. 2009, ApJ, 700, 1456

Conley, A., et al. 2011, ApJS, 192, 1

Contreras, C., et al. 2010, AJ, 139, 519

Cooke, J., et al. 2011, ApJ, 727, L35+

Domínguez, I., Höflich, P., \& Straniero, O. 2001, ApJ, 557, 279

Ellis, R. S., et al. 2008, ApJ, 674, 51

Filippenko, A. V. 1982, PASP, 94, 715

1997, ARA\&A, 35, 309

Filippenko, A. V. 2005a, in Astronomical Society of the Pacific Conference Series, Vol. 332, The Fate of the Most Massive Stars, ed. R. Humphreys \& K. Stanek, 33-+

Filippenko, A. V. 2005b, in Astrophysics and Space Science Library, Vol. 332, White dwarfs: cosmological and galactic probes, ed. E. M. Sion, S. Vennes, \& H. L. Shipman (Dordrecht: Springer), 97-133

Filippenko, A. V., Li, W. D., Treffers, R. R., \& Modjaz, M. 2001, in ASP Conf. Ser. 246: IAU Colloq. 183: Small Telescope

Astronomy on Global Scales, ed. B. Paczynski, W.-P. Chen, \& C. Lemme, 121-+

Foley, R. J., et al. 2012, ApJ, 744, 38

2009, AJ, 138,376

2008a, ApJ, 684, 68

Foley, R. J., Filippenko, A. V., \& Jha, S. W. 2008b, ApJ, 686, 117

Foley, R. J., \& Kasen, D. 2011, ApJ, 729, 55

Foley, R. J., et al. 2003, PASP, 115, 1220

Foley, R. J., Sanders, N. E., \& Kirshner, R. P. 2011, ApJ, 742, 89

Frieman, J. A., et al. 2008, AJ, 135, 338

Fukugita, M., Ichikawa, T., Gunn, J. E., Doi, M., Shimasaku, K., \& Schneider, D. P. 1996, AJ, 111, 1748

Ganeshalingam, M., et al. 2010, ApJS, 190, 418

Gunn, J. E., et al. 1998, AJ, 116, 3040 2006, AJ, 131, 2332

Guy, J., et al. 2007, A\&A, 466, 11

Hamuy, M., et al. 1996, AJ, 112, 2408

Hicken, M., et al. 2009a, ApJ, 700, 331

Hicken, M., Wood-Vasey, W. M., Blondin, S., Challis, P., Jha, S., Kelly, P. L., Rest, A., \& Kirshner, R. P. 2009b, ApJ, 700, 1097

Höflich, P., Wheeler, J. C., \& Thielemann, F.-K. 1998, ApJ, 495, 617
Hogg, D. W., Finkbeiner, D. P., Schlegel, D. J., \& Gunn, J. E. 2001, AJ, 122, 2129

Holtzman, J. A., et al. 2008, AJ, 136, 2306

Horne, K. 1986, PASP, 98, 609

Howell, D. A., Sullivan, M., Conley, A., \& Carlberg, R. 2007, ApJ, 667, L37

Hsiao, E. Y., Conley, A., Howell, D. A., Sullivan, M., Pritchet, C. J., Carlberg, R. G., Nugent, P. E., \& Phillips, M. M. 2007, ApJ, 663, 1187

Ivezić, Ž., et al. 2004, Astronomische Nachrichten, 325, 583

- 2007, AJ, 134, 973

Jha, S., et al. 2006, AJ, 131, 527

Jha, S., Riess, A. G., \& Kirshner, R. P. 2007, ApJ, 659, 122

Kelly, P. L., Hicken, M., Burke, D. L., Mandel, K. S., \& Kirshner, R. P. 2010, ApJ, 715, 743

Kelson, D. D. 2003, PASP, 115, 688

Kessler, R., et al. 2009a, ApJS, 185, 32

. 2009b, PASP, 121, 1028

Konishi, K., et al. 2011, ArXiv e-prints, 1101.1565

Lampeitl, H., et al. 2010a, MNRAS, 401, 2331

- 2010b, ApJ, 722, 566

Leaman, J., Li, W., Chornock, R., \& Filippenko, A. V. 2010, ArXiv e-prints, 1006.4611

Leibundgut, B. 2001, ARA\&A, 39, 67

Lentz, E. J., Baron, E., Branch, D., Hauschildt, P. H., \& Nugent, P. E. 2000, ApJ, 530, 966

Livio, M. 2001, in Supernovae and Gamma-Ray Bursts: the Greatest Explosions since the Big Bang, ed. M. Livio, N. Panagia, \& K. Sahu, 334-355

Marshall, J. L., et al. 2008, in Society of Photo-Optical Instrumentation Engineers (SPIE) Conference Series, Vol. 7014, Society of Photo-Optical Instrumentation Engineers (SPIE) Conference Series

Matheson, T., et al. 2008, AJ, 135, 1598

Miknaitis, G., et al. 2007, ApJ, 666, 674

Miller, J. S., \& Stone, R. P. S. 1993, Lick Obs. Tech. Rep. 66 (Santa Cruz: Lick Obs.)

Nugent, P., Phillips, M., Baron, E., Branch, D., \& Hauschildt, P. 1995, ApJ, 455, L147

Oke, J. B. 1990, AJ, 99, 1621

Oke, J. B., et al. 1995, PASP, 107, 375

Oke, J. B., \& Gunn, J. E. 1983, ApJ, 266, 713

Östman, L., et al. 2011, A\&A, 526, A28+

Perlmutter, S., et al. 1999, ApJ, 517, 565

Perlmutter, S., \& Schmidt, B. P. 2003, in Lecture Notes in Physics, Berlin Springer Verlag, Vol. 598, Supernovae and Gamma-Ray Bursters, ed. K. Weiler, 195-217

Phillips, M. M. 1993, ApJ, 413, L105

Pier, J. R., Munn, J. A., Hindsley, R. B., Hennessy, G. S., Kent, S. M., Lupton, R. H., \& Ivezić, Ž. 2003, AJ, 125, 1559 
Riess, A. G., et al. 1998, AJ, 116, 1009

- 1999, AJ, 117, 707

Riess, A. G., Press, W. H., \& Kirshner, R. P. 1996, ApJ, 473, 88

Riess, A. G., et al. 2007, ApJ, 659, 98

- 2004, ApJ, 607, 665

Sako, M., et al. 2008, AJ, 135, 348

- 2011, ApJ, 738, 162

Sauer, D. N., et al. 2008, MNRAS, 391, 1605

Schlegel, D. J., Finkbeiner, D. P., \& Davis, M. 1998, ApJ, 500, 525

Smith, J. A., et al. 2002, AJ, 123, 2121

Sollerman, J., et al. 2009, ApJ, 703, 1374
Stoughton, C., et al. 2002, AJ, 123, 485

Stritzinger, M. D., et al. 2011, AJ, 142, 156

Sullivan, M., et al. 2010, MNRAS, 406, 782

Sullivan, M., Ellis, R. S., Howell, D. A., Riess, A., Nugent, P. E., \& Gal-Yam, A. 2009, ApJ, 693, L76

Sullivan, M., et al. 2011, ApJ, 737, 102

Tucker, D. L., et al. 2006, Astronomische Nachrichten, 327, 821

Wade, R. A., \& Horne, K. 1988, ApJ, 324, 411

Wood-Vasey, W. M., et al. 2007, ApJ, 666, 694

York, D. G., et al. 2000, AJ, 120, 1579

Zheng, C., et al. 2008, AJ, 135, 1766

\section{APPENDIX}

\section{GALAXY SUBTRACTION}

\section{Photometry Matching and Spectral Warping}

The first method to correct for galaxy contamination makes no assumptions about the quality of our spectrophotometry and is similar to the method presented by Ellis et al. (2008). We perform extractions of our SNe Ia with a fixed aperture and background regions which avoid any host-galaxy light. Our resulting spectra are a combination of SN and host galaxy with no attempt to remove host-galaxy contamination.

As a byproduct of the SMP photometry, we have the broad-band photometry for the host galaxy at the position of the SN. Using multicolor light-curve shape (MLCS2k2; Jha et al. 2007) template light curves, we are able to interpolate the SMP photometry (independently in each band) to determine the broad-band photometry for the SN at the time the spectrum was obtained. Using the photometry of both the SN and the background at the position of the SN, we warp our spectrum to match the measured photometry of the SN and background. We do the warping using both a spline and linear fit with three pivot points at the flux-weighted centers of the gri filters. Since the SEDs are slightly different for each object, we allow the pivot points to shift in wavelength to match the true center of the filter for each spectrum. We can then subtract the reconstructed galaxy SED (properly scaled to the galaxy photometry) from the spectrum, yielding a spectrum which matches the photometry of the SN.

Since a spline will exactly fit the measured photometry, we perform a Monte Carlo simulation to determine if the spline fitting is producing reasonable residual spectra. To do this, we randomly choose photometry for each object assuming Gaussian distributions for the photometry with a mean corresponding to the measured photometry and a width corresponding to the uncertainty in the measurements. Since the uncertainties in the gri bands are of similar magnitude, the average of the residuals from the Monte Carlo simulations is typically very similar to the spline using only the measured photometry.

We find that the photometry is always well fit by a linear function of wavelength resulting in $\chi^{2} /$ dof $\ll 1$, indicating that for many SNe this method is overfitting the data (i.e., no galaxy subtraction is required for some SNe). For most cases the slope of the line is $\lesssim 1 \sigma$ (and in all cases the slope is $<3 \sigma$ ) from 0 , again indicating little to no correction required. For our entire sample, there is no trend in the slope for a given night, standard star, or slit position angle, with nearly equal numbers of SNe with positive and negative slopes, suggesting that there is no systematic problem affecting our galaxy subtraction.

\section{Color Matching}

The second method initially assumes that our spectrophotometry is well calibrated (i.e., errors from the spectrophotometry do not dominate the error in comparing the spectrum to the photometric colors). The flux calibration is verified both by the photometry matching above and also after correcting for host-galaxy contamination. For this method, we attempt to remove as much galaxy contamination as possible when extracting our spectra. Although removing galaxy light at the point of extraction prevents matching to the absolute scaling of photometry, it tries to reduce the introduction of errors by subtracting a potentially imprecise galaxy SED from a spectrum. Since the galaxy photometry determines the reconstructed galaxy SED, if our spectrophotometry is well calibrated, then subtracting the reconstructed galaxy SED from the observed spectrum such that the colors of the residual spectrum matches those of the SN will result in an SN-only spectrum.

We can demonstrate this mathematically as follows. In general, an observed SN spectrum is defined by

$$
f_{\mathrm{spec}}=A\left(f_{\mathrm{SN}}+B f_{\text {gal }}\right) \text {, }
$$

where $f_{\mathrm{spec}}, f_{\mathrm{SN}}$, and $f_{\text {gal }}$ are the vectors of fluxes in the observed spectrum, SN spectrum, and galaxy spectrum, respectively, and $A$ and $B$ are normalization factors. One can think of $A$ as normalizing the spectrum in an absolute sense to account for slit losses, clouds, and other effects. The parameter $B$ can be thought of as a color normalization, where it is adjusted until $f_{\text {spec }}$ and $f_{\mathrm{SN}}+B f_{\text {gal }}$ have the same color.

As a byproduct of the SMP photometry, we have $p_{\text {gal }}$, the broad-band photometry (in flux units) for the host galaxy at the position of the SN. Using multicolor light-curve shape (MLCS2k2; Jha et al. 2007) template light curves, we are able to interpolate the SMP photometry (independently in each band) to determine $p_{\mathrm{SN}}$, the broad-band photometry (in flux units) for the $\mathrm{SN}$ at the time of the spectrum. 
We can define the function which translates spectra to synthetic broad-band photometry as $P$, where $P\left(f_{\mathrm{SN}}\right)=p_{\mathrm{SN}}$ and $P\left(f_{\text {gal }}\right)=p_{\text {gal }}$. Note that we impose the first relationship, while the second relationship is required by our method of determining $f_{\text {gal }}$.

From our spectrum, we are able to determine $P\left(f_{\text {spec }}\right)$, the broad-band synthetic photometry (in flux units) of the spectrum, which includes both SN and galaxy light. These vectors obey the equation

$$
P\left(f_{\text {spec }}\right)=A\left(p_{\mathrm{SN}}+B p_{\text {gal }}\right) \text {. }
$$

For this equation to be valid, we make two assumptions. The first, already noted above, is that our spectra are well flux calibrated. The second assumption is that $B$, the relative fraction of the galaxy and SN light in the observed spectrum, does not vary strongly with wavelength. We will return to both of these assumptions below.

Having gri photometry for the galaxy and the SN, as well as the observed spectrum and the reconstructed galaxy spectrum, we are able to determine the factors $A$ and $B$. We can then use these factors to appropriately remove the reconstructed galaxy spectrum from the SN spectrum,

$$
f_{\mathrm{SN}}=A^{-1} f_{\mathrm{spec}}-B f_{\mathrm{gal}} \text {. }
$$

We have applied this technique to all SN spectra. Figure 1 displays two of our observed spectra, the reconstructed host-galaxy spectra, and the galaxy-contamination corrected SN spectra (see the online version for figures showing all spectra).

\section{Galaxy Subtraction Performance}

We have applied two methods to properly correct for host-galaxy contamination and to recover the SN SEDs for our sample of SNe Ia. One method attempts to match spectra to the measured brightnesses, while the other strives to match to the measured colors. Neither technique is superior for all cases, but we will discuss their performance for our data below.

The greatest advantage of the photometry-matching method is that it can minimize errors from poor spectrophotometry. By definition, the spline-fitting version yields SN SEDs that exactly match the SN photometry, while the line-fitting version will never increase the residuals. However, this method has two main drawbacks. First, it makes no attempt to reduce galaxy contamination during extraction. Second, the warping of the spectrum is somewhat arbitrary and must be extrapolated to wavelengths beyond those covered by our broad-band photometry.

In a situation where a galaxy has a constant SED at all positions and a smoothly varying surface-brightness profile, it is simple to remove the galaxy contamination in a spectrum during extraction. By measuring the galaxy light on either side of the position of the SN in the two-dimensional spectrum and interpolating over the position of the SN, one can cleanly remove all galaxy contamination. If, however, one makes no attempt to remove the galaxy contamination during extraction, but rather relies on a reconstruction of the galaxy SED from broad-band photometry, one will almost certainly introduce additional errors in the spectrum.

If our spectrophotometry is incorrect for a given object, the correct function to warp the spectrum to match the photometry can never be perfectly determined. For our sample, we have assumed that this function is either a spline or a line with anchors at the center of the gri bandpasses with fluxes equal to those measured from the gri photometry. Although the corrections were typically small, deviations from the fit are possible for wavelengths between the anchor points. Additionally, any wavelengths beyond the anchor points must be extrapolated. Since our wavelength coverage $(\sim 3200-9200 \AA)$ is much larger than the span from the bluest to the reddest anchor points (approximately 4800 and $7500 \AA$ for $g$ and $i$, respectively), we must either extrapolate for a significant portion of our spectra or trim our spectra. For the spline fitting, the implied corrections in the extrapolation region can be large, while the corrections from the linear fitting are more realistic. For the spline-fitting version, we have chosen to extrapolate to the blue edge of the $g$ band and the red edge of the $i$ band, $3950 \AA$ and $8220 \AA$, respectively. For these wavelengths, we have some information contributing to the photometry, while also limiting our extrapolation. For the linear-fitting version, we extrapolate further, to $3200 \AA$ and $9000 \AA$.

The one key advantage of the color-matching method is that galaxy subtraction is performed during the extraction of the spectra. This will reduce any error associated with having an incorrect galaxy SED. Again, this method requires two assumptions: the spectrophotometry is not the dominant source of error and the PSF of our object does not vary wildly with wavelength in a way where our parameter $B$ becomes strongly dependent on wavelength.

We can examine our spectrophotometry by comparing synthetic broad-band colors of our spectra to colors from the photometry. In particular, we are most interested in the relative spectrophotometry of our spectra without any attempt to remove galaxy contamination during spectral extraction. These spectra can be directly compared to the photometry of the SN and galaxy at the position of the SN.

In Figure 18, we present the $g-r$ and $r-i$ colors one would find by measuring the fluxes in an aperture centered on the SNe at the time of our spectra with no host-galaxy subtraction in our images, as well as the synthetic colors of our spectra without any attempt to remove galaxy contamination during spectral extraction. These measurements are consistent with $\chi^{2}$ dof $=21.15 / 21$, showing that our spectrophotometry is very good over the bands where we can measure it for these SNe.

A further test of our spectrophotometry is to examine the synthetic photometry of our SN spectra after the galaxy contamination has been removed. In the case of the photometry-matching technique, the residuals are by design small. For the color-matching method, on the other hand, the residuals are not guaranteed to be small. If our 

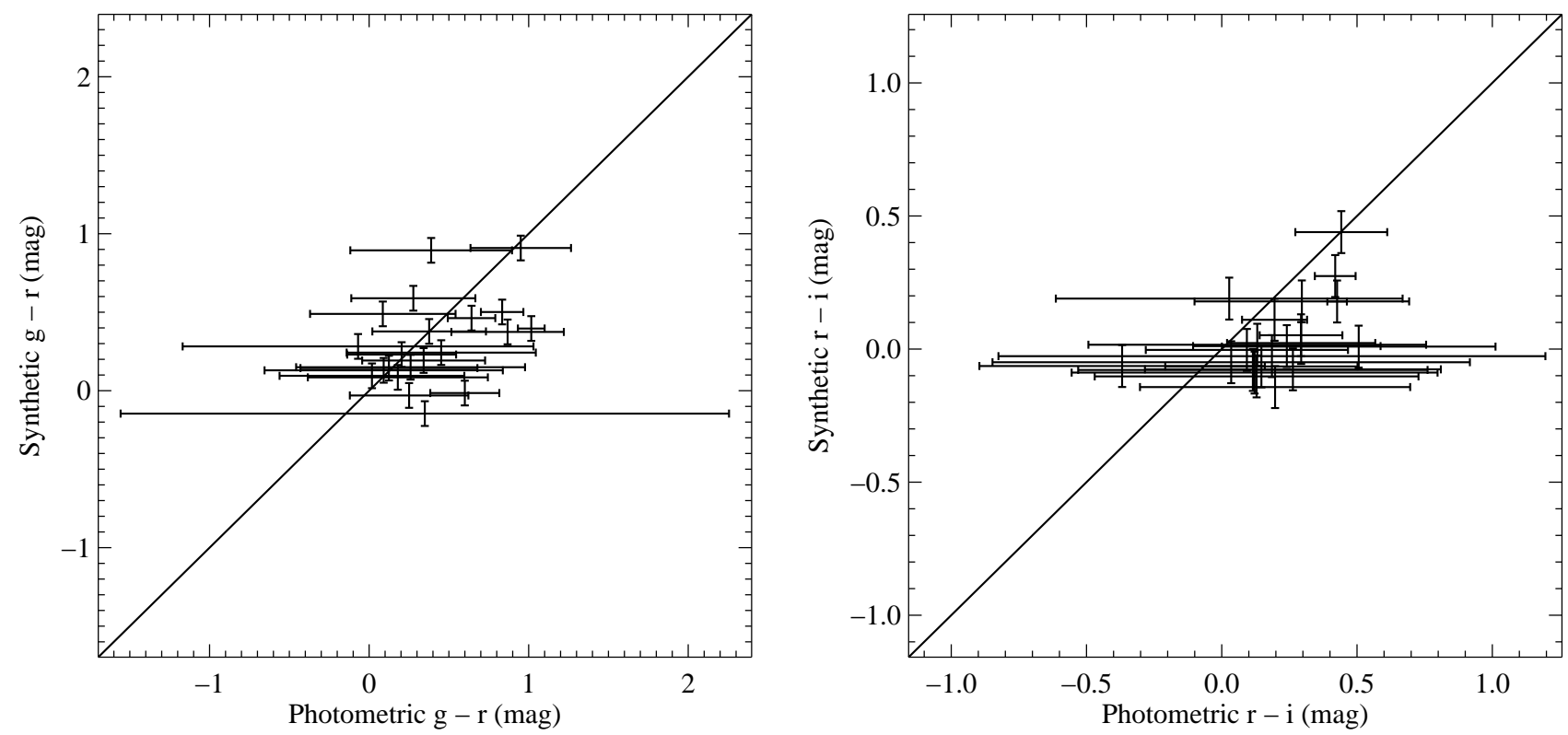

FIG. 18.- (Left) Synthetic $g-r$ colors of our SN spectra with no attempt to remove galaxy contamination compared to the photometric $g-r$ colors determined by adding the photometry of the SN at the time of the spectrum to that of the galaxy at the position of the SN. Overplotted is the line indicating equivalent photometric and synthetic colors. (Right) Synthetic $r-i$ colors of our SN spectra with no attempt to remove galaxy contamination compared to the photometric $r-i$ colors determined by adding the photometry of the SN at the time of the spectrum to that of the galaxy at the position of the SN. Overplotted is the line indicating equivalent photometric and synthetic colors. The assumption that our spectrophotometry is correct yields $\chi^{2} /$ dof $=21.15 / 21$.

spectrophotometry (or alternatively, our galaxy SEDs) were incorrect, we could obtain a minimal residual in the colors that are still far from the measured values for the SN. In Figure [19], we present the ratio of the synthetic fluxes recovered after galaxy subtraction to the measured photometric fluxes for each SN in our sample. Ignoring SNe Ia with low S/N or failed galaxy-template determination, the average of the residuals is $1.03,0.97,1.01$, with a scatter in the residuals of $5.4 \%, 4.5 \%$, and $5.0 \%$ in the $g, r$, and $i$ bands, respectively. If we also remove the SNe with $0.27<z<0.33$, the average of the residuals is $1.03,0.97,1.01$, with a scatter in the residuals of $5.1 \%, 3.7 \%$, and $4.2 \%$ in the $g, r$, and $i$ bands, respectively. These uncertainties are consistent with the errors expected from the spectrophotometry, photometry, and galaxy subtraction (Matheson et al. 2008; Silverman et al., submitted; Blanton et al. 2003). For the photometry-matched, linearly corrected spectra including (excluding) SNe with $0.27<z<0.33$, the average of the residuals is $1.06,1.06$, and $1.08(1.04,1.03$, and 1.07$)$ with scatter in the residuals of $6.0 \%, 9.0 \%$, and $11.8 \%(3.0 \%$, $6.6 \%$, and $9.4 \%$ ) for the $g, r$, and $i$ bands, respectively.

From Figure 19, we can also infer that our parameter $B$ does not vary significantly with wavelength. If, for instance, $B$ were a monotonically increasing function of wavelength, but we were not accounting for it, we would expect to oversubtract our galaxy SED in the $g$ band and undersubtract our galaxy SED in the $r$ band. Since the average values of the ratio of the synthetic to photometric flux are consistent with 1.0 in all bands, we conclude that $B$ is not strongly dependent on wavelength.

From our tests, we find that both the photometry-matching and color-matching methods produce excellent galaxycontamination corrected SN spectra over the gri bands. Because of the extrapolation of the spline function, we do not believe our spline-fit photometry-matched spectra can be extended blueward of the $g$ band or redward of the $i$ band; similarly, the linear-fit photometry-matched spectra are limited to 3200-9000 A. Considering the excellent spectrophotometry of our spectra for the gri bands and our experience with $U$-band flux calibration for many lowredshift SNe Ia (Silverman et al., submitted), the color-matching technique should yield properly flux-calibrated spectra redward of $\sim 3200 \AA$. We have performed our analysis using both the photometry-matched and color-matched spectra, yielding similar results. We will present some results from both methods, but will focus on the color-matched spectra.

\section{Galaxy Subtraction Failures}

An SN fails the galaxy subtraction when the best-fit galaxy SED has larger flux values than the galaxy-contaminated SN spectrum for a significant portion of the spectrum. This can occur if there is incorrect galaxy or SN photometry or if the SN spectrum is not properly flux calibrated. Additionally, for SNe with $0.27<z<0.33$, where incorrect galaxy SEDs are possible, the reconstructed SED may be unphysical.

There are four SNe Ia for which the galaxy subtraction fails. Three of these (SNe 16644, 16789, and 20829) have redshifts of $\sim 0.3$ and significant galaxy contamination. The unphysical SEDs of reconstructed galaxy spectra at $z \approx 0.3$ are the obvious reason for a failure of the fit. Despite this, by constraining a heavily smoothed version of the residual SN spectrum to have non-negative fluxes at all places besides the ends and at emission lines (which may be 


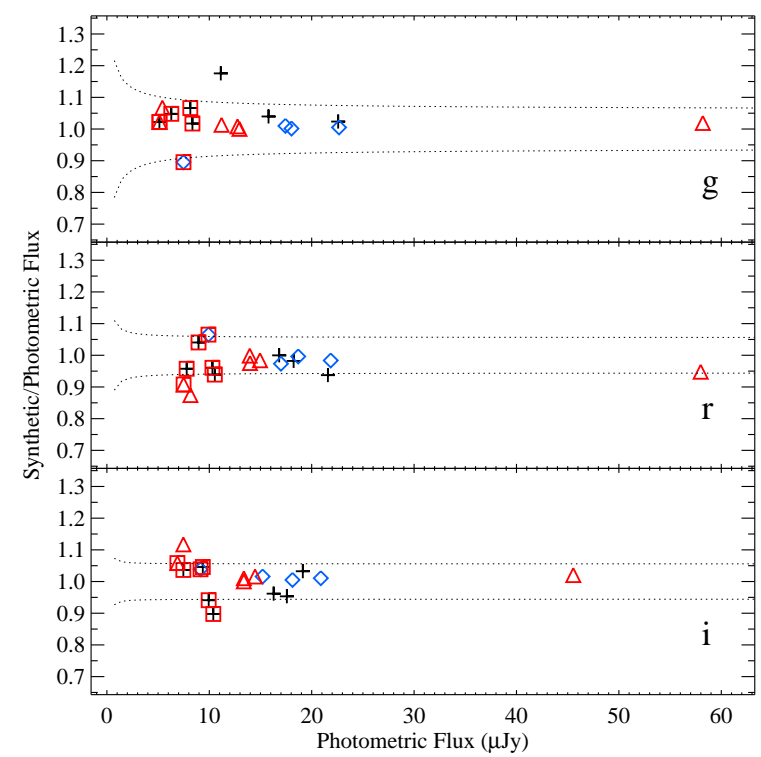

FIG. 19. - Ratio of synthetically created fluxes to measured photometric fluxes for the Nominal sample. The black crosses, blue diamonds, and red triangles correspond to SNe with no, low $(<8 \%)$, and high $(>8 \%)$ host-galaxy contamination, respectively. SNe where the galaxy subtraction did not fail, but are at redshifts $0.27<z<0.33$, have been marked with an additional red square. The dotted lines represent an estimate of the expected error from a combination of photometry, spectral-flux calibration, and galaxy-subtraction errors.

oversubtracted), we can achieve a rough, but probably oversubtracted, SED of the SN. Although this is not a rigorous treatment of the galaxy contamination, it could be useful for visualization.

SN 7512 also fails the galaxy-subtraction procedure. It has relatively low S/N spectra, making galaxy subtraction less precise. The four SNe with failed galaxy subtraction have been removed from our sample for any analysis, leaving a sample of 17 SNe Ia that we use for our analysis. Additional spectroscopy of the SN hosts could improve the SEDs of the SNe with $0.27<z<0.33$, but the faintness of the unobserved host galaxies makes this task difficult with current facilities.

\section{COMPARISON TO SNLS $Z \approx 0.5$ COMPOSITE SPECTRUM}

Two recent studies generated composite spectra of high-redshift SNe Ia. Foley et al. (2008a) presented composite spectra generated from high-redshift SNe Ia from the ESSENCE survey (Miknaitis et al. 2007). Unfortunately, the lack of multi-color information prevented galaxy-contamination correction similar to that implemented in this study. As a result, there was little information on changes to the SN SED. However, a second study by Ellis et al. (2008) of SNLS SNe Ia did present composite spectra after correcting for the galaxy contamination. The two spectra (one "early" spectrum with $t<-4$ days and one "maximum" spectrum with $-4<t<4$ days; both with $\langle z\rangle \approx 0.5)$ can provide an interesting comparison to our composite spectra.

In Figure 20, we illustrate the Keck/SDSS, SNLS, and low-redshift maximum-light composite spectra. For this comparison, we scaled the spectra to the SNLS spectrum over its entire wavelength range. The SNLS spectrum is consistent with both the Keck/SDSS and low-redshift spectra over the wavelength range sampled. The early and maximum-light SNLS spectra both have a median stretch of 1.06 , which corresponds to $\Delta=-0.23$. Therefore, the SNLS SNe have broader light curves (and are correspondingly more luminous SNe Ia) than those of the Keck/SDSS or low-redshift samples. The UV SEDs of SNe Ia are strongly dependent on their light-curve shape (see Section 5.3 ), and since we do not know the average light-curve shape of the composite spectrum (the individual spectra have different weights and therefore contribute different amounts to the composite spectrum), the comparison is of less utility than that of the low-redshift and Keck/SDSS composite spectra. Despite these differences, it is worth noting that the SNLS spectrum has less flux than the Keck/SDSS spectrum (but still within the error region) and slightly more flux than the low-redshift spectrum at most wavelengths (again, still within the error region). Larger differences may exist in these samples, but since the SNLS spectrum does not probe much of the optical region (stopping blueward of $5400 \AA$ ), the lever arm of scaling the spectra to match at long wavelengths is unavailable for this analysis. As seen with the low-redshift and Keck/SDSS spectra in Figure 20, matching the spectra at only these blue wavelengths can cause large divergence at redder wavelengths.

In Figure 21, we present a comparison of the SNLS "Early" composite spectrum with a low-redshift $\langle t\rangle=-6.6$ days composite spectrum from Foley et al. (2008a). Over the restricted wavelength range, the two spectra appear to be consistent. There is a large deviation at $\sim 3100 \AA$, with the SNLS spectrum having excess flux compared to the low-redshift spectrum, but the uncertainties in both the SNLS and low-redshift spectra are large enough for the spectra to be consistent. Similar to the maximum-light spectra, these samples likely have different light-curve shape characteristics, and additional information is necessary to make definitive conclusions. 


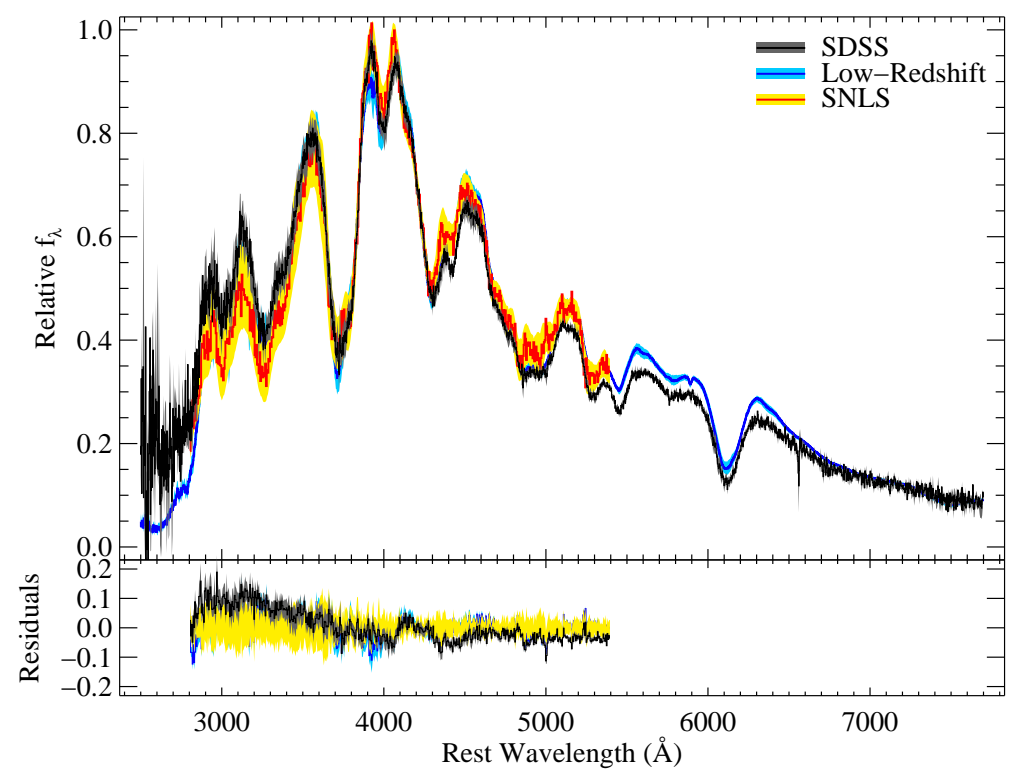

FIG. 20.- (Top panel): Composite spectrum created from our Keck/SDSS sample (black curve) compared to the SNLS maximumlight composite spectrum from Ellis et al. (2008) (red curve), and the maximum-light low-redshift composite spectrum (blue curve). The grey, yellow, and light-blue regions are the $1 \sigma$ boot-strap sampling errors for the Keck/SDSS, SNLS, and low-redshift composite spectra, respectively. (Bottom panel): The yellow region is the $1 \sigma$ boot-strap sampling region for the SNLS composite spectrum. The black and blue curves are the residuals of the SNLS and the Keck/SDSS (Keck/SDSS minus SNLS) and low-redshift (low redshift minus SNLS) composite spectra, respectively. The grey and light-blue regions are the residuals of the SNLS composite spectrum and the Keck/SDSS and low-redshift $1 \sigma$ boot-strap sampling regions, respectively.

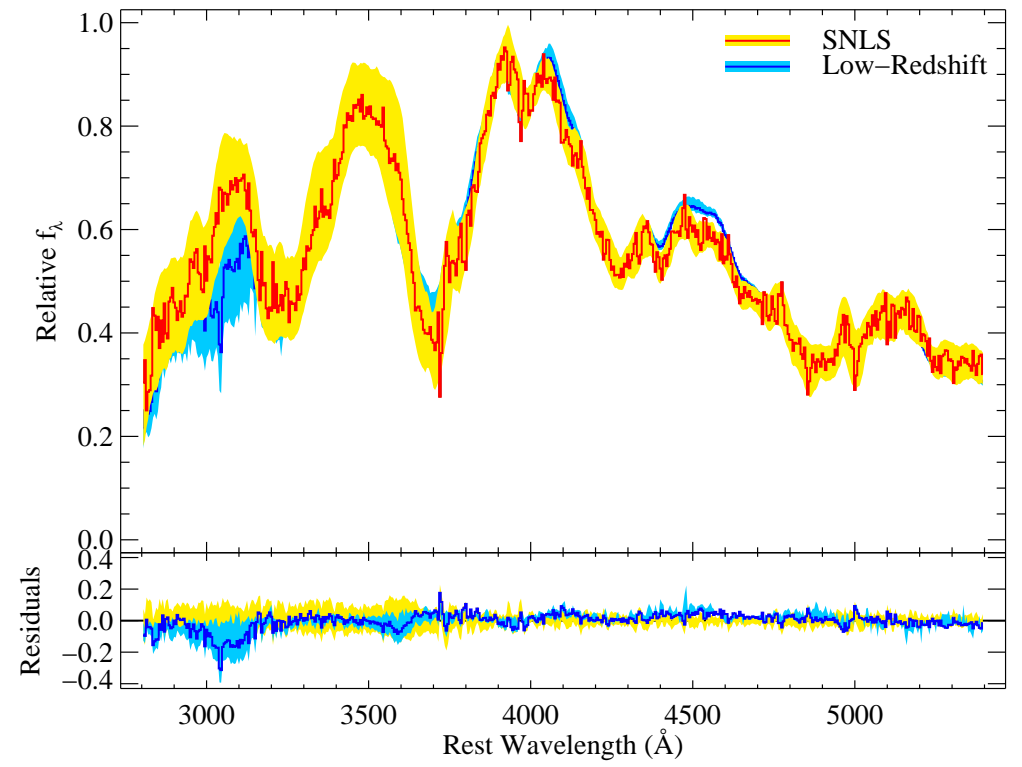

Fig. 21. - (Top panel): SNLS "Early" composite spectrum created from Ellis et al. (2008) (red curve; $t<-4$ days) compared to the premaximum-light low-redshift composite spectrum from Foley et al. (2008a) (blue curve, $\langle t\rangle=-6.6$ days). The yellow and light-blue regions are the $1 \sigma$ boot-strap sampling errors for the SNLS and low-redshift composite spectra, respectively. (Bottom panel): The yellow region is the $1 \sigma$ boot-strap sampling region for the SNLS composite spectrum. The blue curve is the residual of the SNLS and the low-redshift composite spectra. The light-blue region is the residual of the SNLS composite spectrum and the low-redshift $1 \sigma$ boot-strap sampling regions.

The expectation is that any physical change in SN Ia spectra with redshift would be monotonic with redshift. With that in mind, the fact that the SNLS composite spectrum is slightly above the low-redshift composite spectrum and slightly below the Keck/SDSS composite spectrum is puzzling. Perhaps the Keck/SDSS sample is biased in a way we have not been able to detect, or our assumption of monotonic spectral behavior is incorrect. However, it is worth noting that when splitting the Keck/SDSS sample by redshift, the higher-redshift composite spectrum has less UV flux than the lower-redshift composite spectrum. Although this difference is not significant given our boot-strap sampling 
errors, it shows the same trend as with the SNLS spectra.

Without combining the individual SNLS SN Ia spectra in the same manner as the Keck/SDSS SN Ia spectra, it is difficult to quantify the similarities and differences. In particular, Ellis et al. (2008) warped the spectra to match their photometry before correcting for host-galaxy contamination. The reasoning for this is that differential slit losses and atmospheric extinction cause errors in the spectrophotometry. We have shown that our reduction methods reproduce $B-V$ colors with a low dispersion $(\sigma=0.063 \mathrm{mag})$ for low-redshift SNe Ia (Matheson et al. 2008), and we see that there is a low scatter in the synthesized photometry (Figure 19) of our sample of objects with no discernible host galaxy in the images (despite there possibly being some underlying host contributing slightly to the spectrum). Hence, we conclude that our spectrophotometry is reasonable and has uncertainties similar to those associated with host-galaxy contamination. Furthermore, the spectral warping of Ellis et al. (2008) extrapolates into the rest-frame UV for the lower-redshift $(z \lesssim 0.3)$ objects. 